\title{
A two-phase model of skin epidermal stratification: lessons from centrosomes
}

Mareike Damen ${ }^{1,2,3}$, Lisa Wirtz ${ }^{1,2,3}$, Ekaterina Soroka ${ }^{1,2}$, Houda Khatif $^{1,2}$, Christian Kukat ${ }^{4}$,

Benjamin D. Simons ${ }^{5}$, Hisham Bazzi ${ }^{1,2, *}$

${ }^{1}$ Department of Dermatology and Venereology, University Hospital of Cologne, University of

Cologne, 50931 Cologne, Germany

${ }^{2}$ The Cologne Cluster of Excellence in Cellular Stress Responses in Aging-associated Diseases

(CECAD), University of Cologne, 50931 Cologne, Germany

${ }^{3}$ Graduate School for Biological Sciences, University of Cologne, 50674 Cologne, Germany

${ }^{4}$ FACS \& Imaging Core Facility, Max Planck Institute for Biology of Aging, 50931 Cologne,

Germany

${ }^{5}$ The Welcome Trust/Cancer Research UK Gurdon Institute, University of Cambridge,

Cambridge CB2 1QN, UK

*Correspondence: hisham.bazzi@uk-koeln.de (H.B.)

Key words: delamination, centriole, cilia, p53, 53BP1, USP28 


\section{Abstract}

The development of complex stratified epithelial barriers in mammals is initiated from singlelayered epithelia. How stratification is initiated and fueled are still open questions. Previous studies on skin epidermal stratification suggested a central role for perpendicular/asymmetric cell division orientation of the basal keratinocyte progenitors. Here, we use centrosomes, that organize the mitotic spindle, to test whether cell division orientation and stratification are linked. Genetically ablating centrosomes from the developing epidermis led to the activation of the p53-, 53BP1- and USP28-dependent mitotic surveillance pathway causing a thinner epidermis and hair follicle arrest. The centrosome/p53-double mutant keratinocyte progenitors significantly altered their division orientation in the later stages without majorly affecting epidermal differentiation. Together with time-lapse imaging and tissue growth dynamics measurements, the data suggested that the first and major phase of epidermal stratification is boosted by high proliferation rates of the basal and suprabasal transit-amplifying cells as well as cell delamination, whereas the second phase maybe uncoupled from the division orientation of the basal progenitors. The data provide insights for tissue homeostasis and hyperproliferative diseases that may recapitulate developmental programs. 


\section{Introduction}

The generation of complex stratified epithelia, including the skin epidermis, during mammalian embryonic development is essential to form barriers that are compatible with postnatal life ${ }^{1}$. The stratified skin epidermis is initiated from the single-layered simple epithelium, derived from the ectoderm, surrounding the embryo ${ }^{2}$. Around embryonic day (E) 9.5, the epithelial cells commit to stratification through the master regulator p63, a member of the p53 family of transcription factors ${ }^{3,4}$. The progenitor cells down-regulate the expression of the simpleepithelial keratin intermediate filaments, such as Keratin-8 (K8), and express the complexepithelial keratins, $\mathrm{K} 5$ and $\mathrm{K} 14$. The periderm is the first layer generated and it acts as a transient protective and insulating barrier for the developing embryo ${ }^{5}$. Around E12.5, the first differentiated suprabasal epidermal layer cells appear and are characterized by the expression of a distinct set of keratins, $\mathrm{K} 1$ and $\mathrm{K} 10^{6}$. Subsequently, the epidermal keratinocytes undergo further differentiation and crosslinking to generate a fully functional barrier by $\mathrm{E} 17.5^{7}$. The stereotypical regeneration and differentiation program in the epidermis, starting from the basal stem cells, to replenish the shed corneocytes persists throughout the life of the animal ${ }^{8}$. In addition to the interfollicular epidermal stem cells, the basal keratinocyte progenitors also give rise to all the stem cells of the skin epithelium, including the hair follicle stem cells ${ }^{9}$.

The mechanism of how the new layers of the stratified epidermis are generated and maintained are not well-understood. The published data support a model for interfollicular epidermal stratification that is coupled to the orientation of cell division in the basal layer of the epidermis 2,10. At E12.5 and earlier, almost all of the progenitor basal cells divide with an axis that is parallel to the basement membrane and undergo a presumptive symmetric division to generate two progenitor daughter cells that remain in the basal layer ${ }^{11}$. From E13.5 onwards, more of the dividing basal progenitors shift their axis of division to a perpendicular orientation, which has become synonymous with an asymmetric division, to generate one daughter progenitor cell that 
remains in the basal layer, and one differentiated daughter cell that stratifies the forming epidermis ${ }^{11,12}$. Several studies in the literature are consistent and correlate with the division orientation-based model ${ }^{13-19}$. For example, manipulations in gene products that result in a thinner epidermis, such as knockdown of LGN or NuMa1, are highly associated with an increase in the fraction of parallel divisions, whereas those causing a thickened hyperdifferentiated epidermis, such as overexpression of INSC, are correlated with an increase in the fraction of perpendicular divisions ${ }^{12}$. Importantly, there is currently no consensus in the field on what stage of epidermal development, for example E14-E15 versus E16-E17, or phase of mitosis, in particular metaphase versus late anaphase/telophase, should be considered during cell division orientation analyses; however the majority suggests the later developmental stage as well as late mitotic phase ${ }^{13-19}$. In this study, we use centrosome loss-of-function to test the causal relationship between progenitor division orientation and epidermal stratification during the later stages of epidermal development.

Centrosomes are major microtubule-organizing centers of animal cells that are composed of a pair of centrioles surrounded by a proteinaceous matrix ${ }^{20}$. Centrosomes are essential to provide the centriolar template for cilia, and are important for efficient mitotic spindle assembly ${ }^{21}$. In humans, mutations in genes encoding centrosomal proteins lead to primordial dwarfism and microcephaly ${ }^{22}$. We have previously shown that the constitutive ablation of Sas-4, a gene essential for centriole formation and duplication, led to the loss of centrioles and cilia in early developing mouse embryos ${ }^{23}$. The loss of centrioles, but not the secondary loss of cilia, resulted in p53-dependent cell death and embryonic arrest at E9.5 ${ }^{23}$. Conditional ablation of Sas-4 in the developing brain recapitulated the human microcephaly phenotype and led to p53dependent cell death of the radial glial progenitors (RGPs) in the cortex ${ }^{24}$. Activation of this p53-dependent pathway was independent of DNA damage or chromosome segregation errors, and instead was associated with prolonged mitotic duration ${ }^{23,24}$. Recent reports in cultured 
mammalian cell lines have confirmed our findings and extended them to include 53BP1 and USP28 as new components acting upstream of p53 in a novel pathway now termed "the mitotic surveillance pathway" ${ }^{25-30}$.

Published data on brain development suggested that neurogenesis in the brain cortex depends on the regulation of cell division orientation of the RGPs in the ventricular zone of the cortex ${ }^{31}$. However, the Sas-4 p53 double mutant RGPs showed randomized cell division orientation relative to controls, yet resulted in relatively normal cortical development and layering ${ }^{24}$. In this work, we conditionally removed Sas-4/centrioles and p53 from the developing skin epidermis to test whether the keratinocyte basal progenitors can uncouple cell division orientation from epidermal stratification and differentiation. To separate the functions of centrioles in cilia formation versus spindle assembly, we also conditionally removed Ift88, a gene required for the formation of cilia but not centrioles ${ }^{32-34}$. Our data showed that the loss of centrioles, but not cilia, resulted in early p53-dependent cell death, leading to a thinner epidermis and arrested hair follicles. These phenotypes were rescued in the Sas-4 p53 double mutant epidermis, which resembled controls and cilia mutants. Importantly, the double mutant basal keratinocyte progenitors showed a significant shift in cell division orientation that was uncoupled from epidermal stratification and differentiation at the later stages of epidermal development. Using time-lapse imaging that utilizes a new skin explant method and measurements of tissue growth dynamics in developing embryos, the data supported a revised two-phase model of epidermal stratification and development that highlights the importance of the early phase (E13-E15) and is based on cell delamination and high proliferation in basal and suprabasal progenitors. 


\section{Results}

\section{Centrioles are important, but not essential, for mouse epidermal and hair follicle} development

In order to assess the functions of mammalian centrioles and centrosomes in the developing mouse skin epithelium, we deleted Sas-4 using a K14-Cre line ${ }^{35}$, which expresses the Cre recombinase in developing stratified epithelia, including the skin epidermis as early as embryonic day (E) 9.5, in combination with a Sas-4 conditional allele ${ }^{23,24}$. Immunostaining for $\gamma^{-}$ Tubulin (TUBG, a marker for centrosomes) and CEP164 (a marker for centriolar distal appendages) confirmed that centrioles are almost completely lost from the epidermal basal and suprabasal cells by E15.5 (Fig. 1a-c). Consistent with the reduced viability of the centrosome mutant animals by P3 ( 60\% compared to $\sim 95 \%$ for control animals), the embryos at E17.5 showed a slight delay in skin barrier formation at the chin and the paws, as judged by a Toluidine Blue dye-penetration assay, which was restored just before birth at E18.5 (Fig. 1d). At postnatal day 21 (P21), the centrosome mutant mice were smaller than their control littermates and had a grossly thin and transparent skin with very sparse hair (Fig. 1e; Fig. S1a). Starting as early as $\mathrm{P} 4$, the centrosome mutant mice had a significantly reduced weight relative to their littermates (Fig. S1a), likely due to the K14-Cre Sas-4 defects in the oral epithelium which impaired food intake. To avoid any secondary complications of centriole loss after birth, we focused most of our analyses on embryonic development until PO.

At P0, the Sas-4 mutant epidermis was significantly thinner than that of control littermates with a marked reduction in the number of hair follicles (Fig. 1e-g). To study if the epidermal differentiation process was affected upon the loss of centrioles, we performed immunostainings on back-skin sections at P0. Staining for markers of the proliferative basal layer (K14), and differentiated layers (K1 and Loricrin, LOR), showed that although the epidermis and its differentiated layers were thinner, the differentiation markers were not majorly affected in the 
Sas-4/centrosome mutant epidermis (Fig. 1h). However, K6A, which is ectopically expressed in activated keratinocytes and in skin disease conditions, was markedly increased and indicated an abnormal response in the Sas-4 mutant skin epithelium (Fig. 1h).

To test whether the secondary loss of cilia upon the removal of centrioles caused the centrosome-mutant phenotypes, we used the K14-Cre line and a conditional allele of Ift88 ${ }^{32-34}$. Immunostaining for TUBG and ARL13B (a marker for cilia) showed the presence of centrosomes but the loss of cilia in the Ift88 mutant epidermis at E15.5 (Fig. 1a,b). Compared to Sas4/centrosome mutants, we did not observe major skin phenotypes in the epidermis and hair follicles of Ift88/cilia mutants, as judged by morphological, histological and immunofluorescence examination (Fig. 1e-h; Fig. S1b,c), indicating that the centrosome mutant phenotypes are not due to the loss of cilia.

We concluded that, in contrast to cilia, centrioles and centrosomes are important to ensure normal development of the skin epithelium; however, despite the centriole deficiency in the basal keratinocyte progenitors, they showed robust regulation to allow the formation and maintenance of a generally functional skin barrier.

\section{The loss of centrioles in the epidermis leads to p53 upregulation and transient cell death}

Next, we used immunostaining to assess the molecular consequences of the loss of centrioles during epidermal development. The data showed that there was a gradual upregulation of nuclear p53 from E12.5-P0 in the Sas-4/centrosome mutant basal epidermis (Fig. 2a,b). In contrast, no upregulation of p53 was observed in the Ift88/cilia mutant epidermis at E15.5 (Fig. S2a), indicating that cilia loss alone did not activate the same pathway ${ }^{23,24}$. In addition, cell death, marked by Cleaved-Caspase 3 (CI.CASP3), was significantly increased in the Sas-4 mutant epidermis at E15.5 (Fig. 2c,d). At P0, cell death was rarely detected while p53 was still 
high in the basal keratinocytes of centrosome mutants compared to controls (Fig. 2a,b,e). To check whether the high p53 in the centrosome mutant keratinocytes at P0 caused cell cycle arrest in G1 instead of cell death, similar to the reports in mammalian cell lines that lose centrioles in vitro ${ }^{25,26,36}$, we performed cell cycle analyses on isolated epidermal keratinocytes. The cell cycle profiles showed no significant increase in the G1 population in centrosome mutant keratinocytes compared to controls (Fig. 2f).

Centriole loss causes prolonged mitotic duration which increases the mitotic index ${ }^{23}$. We quantified the number of mitotic cells, marked by phospho-Histone $\mathrm{H} 3(\mathrm{pHH} 3)$, in the basal layer of the epidermis of centrosome mutants and controls at E15.5 (Fig. 2g). We observed an increase in the mitotic index in the centrosome mutant epidermis compared to the control littermates (Fig. $2 \mathrm{~g})^{23,24}$. The delay in mitosis in centrosome mutants was also reflected in a slight, but not significant, increase in the G2/M keratinocyte population at P0 (Fig. 2f), likely because mitosis constitutes only a minor fraction of the total cell cycle $(\sim 3 \%)$.

To gain a deeper understanding of the transcriptional changes in epidermal keratinocytes upon centriole loss, we performed RNA-Seq analyses on micro-dissected and enzymatically separated back-skin epidermis of control and Sas-4 mutants at E13.5, before any major phenotypes were apparent. Among the 258 differentially expressed genes, the vast majority $(80 \%)$ were upregulated in centrosome mutant keratinocytes (Table S1). As expected, gene ontology analyses showed that the most significantly over-represented category was the "p53 downstream" signaling pathway (Fig. 2h). Consistent with the increased cell death in these mutants (Fig. 2c,d), the p53-downstream apoptosis target genes Bbc3 (PUMA) and Pmaip1 (NOXA) as well as the pro-apoptotic FAS ligand and BAX, were among the upregulated genes (Table S1). In addition, p63 and p73 signaling pathways were over-represented among the 
upregulated genes, suggesting that the upregulated p53 in centrosome mutants might interfere with these closely-related family members (Fig. $2 \mathrm{~h}$ ).

To assess the later transcriptional changes caused by p53 upregulation in the centrosome mutant keratinocytes, we performed RNA-Seq on isolated keratinocytes from centrosome mutants and controls at P0. The data showed that over 3600 genes were differentially expressed at this stage, $\sim 40 \%$ of which were upregulated in centrosome mutants (Table S2). Besides the p53-downstream, p73 and p63 pathways, the EGFR (ErbB) and HIF1 pathways were also overrepresented (Fig. S2i). In agreement with K6A upregulation (Fig. 1h), Krt6a and Krt6b were among the most highly induced genes in centrosome mutants, together with many epidermal differentiation-related genes (S100a and Sprr genes) (Table S2). Cdkn1a (p21), which is induced upon the activation of the mitotic surveillance pathway in vitro, was also among the upregulated genes, and was evident by immunostaining in the centrosome mutant epidermis at P0 (Fig. S2b). Both RNA-Seq experiments highlighted the perturbation in the p53/p63/p73 signaling axis in centrosome mutant keratinocytes, where more than $30 \%$ of the differentiallyexpressed genes at E13.5 and $\sim 20 \%$ at P0 have been shown to be targets of p63 in keratinocytes (Fig. 2j) ${ }^{37}$. Similar to controls, p63 and p73 were still readily detectable by immunostaining in the nuclei of basal cells in the centrosome mutant epidermis at P0 (Fig. S2c), suggesting that the unusually high levels of p53 in the nuclei of centrosome mutant keratinocytes might compete with p63 and p73 for target binding.

\section{Centrosome mutants activate the mitotic surveillance pathway}

Centrioles are required for efficient mitosis, which is associated with the suppression of the activation of the p53-dependent mitotic surveillance pathway (Fig. 3a) ${ }^{30}$. We tested whether the loss of p53 can bypass the activation of this pathway in the centrosome mutant skin epidermis (Fig. 3b). We observed that the simultaneous knockout of $p 53$ in the Sas-4/centrosome mutant 
skin epidermis significantly rescued the gross epidermal and hair follicle defects, including the epidermal thickness and hair follicle numbers (Fig. 3b-d). Moreover, the increase in K6A expression in centrosome mutant keratinocytes was dependent on p53, because the Sas-4 p53 double mutant epidermis showed a rescue of K6A back to the levels in the control (Fig. 3e). However, the loss of p53 does not rescue cilia formation or prolonged mitosis, because it does not restore centrioles or shorten mitosis ${ }^{23}$, and thus, the double mutants still showed a higher mitotic index compared to controls (Fig. 2g).

We then asked whether the loss of Usp28 or 53bp1, which have been implicated in the activation of the mitotic surveillance pathway in human cell lines in vitro (Fig. 3a) ${ }^{27-29}$, would recapitulate the p53 deficiency in centrosome mutants in vivo. We generated Usp28 and 53bp1 mutant mice using CRISPR/Cas9 (see Materials and Methods) and crossed each allele to the Sas-4 conditional mutants and K14-Cre to generate Sas-4 Usp28 and Sas-4 53bp1 double mutant skin epidermis. Remarkably, our data showed that these mice had a phenotypic rescue similar to the Sas-4 p53 double mutants, establishing their essential role in the p53-dependent pathway in vivo (Fig. 3b-d). These rescues were quite significant even though our CRISPR/Cas9-generated mutations in Usp28 and 53bp1 had residual proteins by immunostaining (Fig. S3a,b), suggesting that they were likely to be hypomorphic alleles. Consistent with the variable nature of hypomorphic mutations, p53 nuclear levels only significantly decreased in the Sas-4 53bp1 double mutants compared to the Sas-4 single mutants (Fig. S3d).

\section{Cell division orientation of the basal progenitors does not correlate with epidermal differentiation in the mutants}

The Sas-4/centrosome p53 double mutants, which showed normal epidermal thickness at birth (Fig. 3b,c), allowed us to examine the consequences of the loss of centrioles in regulating cell 
division orientation and its relationship to epidermal differentiation. We used immunostaining for Integrin-a6 (ITGA6), to mark the basement membrane zone, and Survivin, a mid-body marker to highlight the mitotic cells in late anaphase to telophase when the division orientation is final, and measured the angles of cell division orientation in sections of the back-skin of Sas-4 p53 double mutant and control animals (Fig. 4a-d) ${ }^{12}$. Published data that analyzed late stages of mitosis considered E16-E17 as the peak of epidermal stratification, with a fine balance between parallel $\left(0-29^{\circ}\right)$ and perpendicular $\left(60-90^{\circ}\right)$ division orientation, whereas earlier stages (E14-E15) showed random orientation proportions ${ }^{18}$. Consistent with this, at E15.5, there was no significant difference between the cell division orientation proportions between control and Sas-4 p53 mutants (Fig. 4b). Statistical analyses (two-way ANOVA and Kolmogorov-Smirnov test) showed that the three division orientation bins (parallel, oblique or perpendicular) had an equal likelihood of occurrence regardless of the genotype, suggesting that they are random in nature.

However, at E16.5 and E17.5, the measurements showed a significant shift towards more perpendicular cell division orientation in the Sas-4 p53 double mutant skin epidermis $(\sim 60 \%)$ at both E16.5 and E17.5 compared to heterozygous and p53 mutant controls ( $40 \%$ ) (Fig. 4c,d; Fig. S4a). The increase in the proportion of perpendicular division orientation in the mutants was accompanied by a significant decrease in parallel division orientation at both stages $(\sim 15-20 \%$ in the mutants compared to $\sim 40 \%$ or more in controls) (Fig. 4c,d). In contrast, similar measurements in Sas-4 single mutants at E16.5, which have a thinner epidermis by P0 (Fig. 1f), had an increased proportion of parallel divisions (Fig. S4b), in line with the prevailing model (see Discussion).

We next tested if the cell division orientation data in Sas-4 p53 double mutants at E16-E17 were consistent with the current model of basal keratinocyte progenitors' division orientation determining the balance between proliferation and differentiation. First, if a perpendicular 
division orientation was asymmetric in nature, then an increase in its proportion should lead to more differentiation in the mutants. Thus, we measured the thickness of the K1-positive suprabasal layers at E16.5, and the data showed that it was not changed in the mutants relative to controls (Fig. 4e,f). In addition, the skin epidermal histology and thickness in the Sas-4 p53 double mutant skin at birth were also similar to controls (Fig. 3b,c). Second, if a parallel division orientation was symmetric, then a decrease in its proportion should result in a lower density of basal keratinocyte progenitors. Instead, the Sas-4 p53 double mutant skin epidermis showed an increase in the density of basal, as well as suprabasal, cells compared to controls (Fig. 4g,h). Accordingly, the average cell size in each layer decreased proportionally in the double mutants (Fig. 4i,j). Both phenotypes of cell number and average size changes in the Sas4 p53 double mutants seemed to be dependent on p53 and/or cilia (Fig. S4c,d). We then checked whether increased proliferation could explain the maintenance, or even increase, in the number of basal cells in the mutants, but the data using EdU incorporation showed no significant difference between the mutants and controls (Fig. S4e). In this context, our data suggested that basal keratinocyte progenitors' proliferation and differentiation were uncoupled from cell division orientation during the later stages of epidermal skin development.

\section{Cell delamination may contribute to epidermal stratification}

Because our data suggested that basal progenitor division orientation may not necessarily dictate epidermal stratification and differentiation, we next asked whether other mechanisms, such as cellular delamination, contribute to stratification during development. Embryonic development on the organismal level is reliant on proliferation and cell division in vivo.

Therefore, we used a well-established ex vivo developing mouse skin culture which recapitulates epidermal differentiation as well as hair follicle development ${ }^{38}$. We treated skin explant cultures at E13.5, which had only a thin K10-positive differentiated layer, with Mitomycin C (MMC) to stop proliferation and cell division, and then incubated the explants for one (E14.5) or two (E15.5) 
days ${ }^{39}$. Proliferation and cell division were drastically inhibited in the MMC-treated skin explants compared to controls, as assayed by EdU incorporation and $\mathrm{pHH} 3$ staining at E14.5 and E15.5 (Fig. S5a). We observed some speckle nuclear staining of pHH3 in MMC-treated skin, presumably in cells arrested in G2, but no staining reflecting condensed mitotic chromosomes. Importantly, despite the inhibition cell division and elevated cell death (Cl.CASP3, Fig. S5b), the MMC-treated skin epidermis retained some capacity to stratify and differentiate, as shown by the substantial increase in the K10-positive layer thickness at E14.5 and E15.5 (Fig. S5a,c). Moreover, the number of basal (K10-negative) cells sharply decreased in the MMC-treated explants compared to the corresponding controls at E14.5 and E15.5 (Fig. S5d). In addition, the number of K10-expressing suprabasal cells in the MMC-treated explants increased $\sim$ four-fold at E14.5 and E15.5 compared to the E13.5 controls, but was still significantly lower than that in the vehicle-treated controls (Fig. S5e). The combined cell number deficiency in the MMC-treated skin cultures was not unexpected because of the inhibition of proliferation and resulting cell death (Fig. S5a,b). The data suggested that upon the inhibition of proliferation and cell division, other mechanisms, most likely cellular delamination from the basal to the suprabasal layers, can contribute to epidermal stratification and differentiation ${ }^{18}$.

\section{Time-lapse imaging reveals an epidermal basal layer flexibility, cellular delamination and}

\section{transit-amplifying divisions}

We then asked whether a perpendicular cell division orientation and/or cellular delamination of the basal progenitors contribute to epidermal stratification and the formation of the suprabasal layers. We recorded time-lapse movies of the skin explants on filters between E13.5-E15.5 ${ }^{40}$; however, the Z-dimension resolution was not sufficient to follow dividing cells (Fig. S5f). Thus, we flipped the explants as skin rolls to transform the initial Z-dimension into an XY-plane (see

Materials and Methods). The new culture method did not affect skin epidermal stratification and differentiation as shown by $\mathrm{K} 10$ staining, and even better resembled the embryonic skin in vivo 
than the flat skin cultures (compare the staining on skin rolls in Fig. S5g and the flat skin explants in Fig. S5h). We focused on the daughter cells of perpendicularly dividing basal progenitors $\left(\sim 80-90^{\circ}\right)$ that point away from the epidermal-dermal interface, and assessed their position over time. Our data showed that almost half of these cells could be incorporated close to the neighboring basal cells (Fig. 5a; Movie S1), whereas the other half remained in the "second" layer (Fig. 5b; Movie S2). Moreover, we observed progenitor basal cells that delaminated, moved up and divided suprabasally shortly afterwards (Fig. 5c; Movie S3). The data from the time-lapse experiments suggested that the progenitors in the basal layer can stratify by delamination and may be boosted by suprabasal divisions to form the multi-layered epidermis, most likely independent of cell division orientation.

During our analyses of the developing mouse embryonic skin epidermis, we noticed that the basal layer character does not necessarily require that the basal keratinocytes appear in direct contact with the underlying basement membrane. This was particularly evident upon staining with ITGA6 (basal) and K10 (suprabasal) at different epidermal developmental stages (Fig. 5d). From E13.5-E17.5, ITGA6 was surrounding the entire basal keratinocytes, including some that seemed to be in the "second" layer but were negative for K10 (Fig. 5d), as well as the daughter cells in a perpendicular division that point away from the epidermal-dermal interface (Fig. 4a) ${ }^{41}$. The ex vivo flat skin explant culture on filters at E13.5, which was just released from the stretching tension of the embryo, showed an even thicker ( three cell layers) ITGA6-positive and K10-negative pseudo-stratified basal layer at E13.5 (Fig. S5h), supporting the in vivo data. On the other hand, basement membrane markers, such as COL4A4 and LAMA1, were confined to the basement membrane at E16.5 (Fig. S5i,j). Collectively, our data suggested that the skin epidermal basal layer is more flexible during development where cells move to a seeminglysecond layer, for example during a perpendicular division, but still retain a basal keratinocyte progenitor character. 


\section{The developing epidermis stratifies and differentiates in two phases}

To gain more insight into the epidermal stratification process at the different stages, we turned to a modelling-based approach (Supplementary Theory in Materials and Methods) informed by measurements of dynamic tissue growth parameters during back-skin interfollicular epidermal development (E12.5-E18.5), in the antero-posterior axis (sagittal sections, Fig. 6) and the dorsoventral axis (transverse sections, Fig. S6). We quantified the following parameters: 1) the net rate of embryonic growth (Fig. 6a; Fig. S6a,b), 2) the density of basal and suprabasal cells (Fig. 6b,c; Fig. S6c,d), 3) the proliferation index of basal and suprabasal cells (Fig. 6d; Fig. S6e). Based on our data above (Fig. 5d), we defined basal progenitor cells by their expression of ITGA6 but not K10, whereas suprabasal cells express K10 but not ITGA6. While the total embryonic growth was approximately linear along the different axes between E12.5-E18.5 (Fig. 6A; Fig. S6a,b), we made two important observations regarding the suprabasal layer. First, the major increase in the number of suprabasal cells occurred between E13.5-E15.5 (Fig. 6b,c; Fig. S6c,d). Second, the suprabasal cells were highly proliferative during the same window of epidermal development (Fig. 6d; Fig. S6e), consistent with the data from the time-lapse imaging of skin roll explants (Fig. 5c; Movie S3) ${ }^{10,42}$. Overall, our measurements and modelling support a two-phase behavior for basal and suprabasal cells during epidermal stratification and differentiation (Fig. 6e). The first phase between E12.5-E15.5 is characterized by proliferation and amplification to fuel stratification, where the suprabasal cells act as a transit-amplifying population that proliferates to populate the newly-forming suprabasal layers. Consistently, the total number of cells rises exponentially by a factor of 7.4 during this phase (Fig. 6e), almost twice the net area expansion of the tissue (Fig. 6a; Fig. S6a,b). In addition, the density of suprabasal cells reaches parity and equilibrium with that of the basal progenitor cells at the end of this phase (at E15.5; Fig. 6c; Fig. S6d). The second phase between E15.5-E18.5 is characterized by a precipitous slow-down in the proliferation rate, especially in the suprabasal 
cells which largely undergo terminal differentiation (Fig. 6d; Fig. S6e). The total number of cells in this second phase rises linearly by a mere factor of 2 (Fig. 6e), in proportion to the net expansion of the tissue. The cell densities in both layers remain similar and largely unchanged during this phase (Fig. 6c; Fig. S6d). Collectively, our data suggest that the major phase of epidermal stratification takes place between E13.5-E15.5 and is boosted by the proliferating and transit-amplifying suprabasal cells ${ }^{10,42}$.

\section{The human skin epidermis harbors a transit-amplifying suprabasal population that is activated in hyperproliferative disorders}

Our data on the high proliferation rate of suprabasally-committed keratinocytes prompted us to further investigate whether the adult human skin also harbors such a population and assess if it is more prominent in hyperproliferative disorders of the skin ${ }^{43-45}$. Immunostaining using the proliferation marker Ki67 together with $\mathrm{K} 10$ showed that 3\% of the K10-positive suprabasal cells in the normal human skin were proliferative (Fig. 7a). As expected in hyperproliferative skin diseases, such a psoriasis and atopic dermatitis, the percentage of proliferative cells was increased $\sim$ four-fold in the basal layer, and so did that of the suprabasally-proliferative population, which increased $\sim$ two-fold (Fig. 7a). The data indicated that the human epidermis has a transit-amplifying population that is increased in certain disease conditions. 


\section{Discussion}

How simple epithelia transform into complex stratified barriers, and whether the stratification and differentiation programs are dependent on a shift to a perpendicular division orientation of the progenitors, are still open questions in epithelial biology. Here, we use centrosome mutants to disrupt cell division orientation and test its relationship with skin epidermal differentiation.

In the developing Sas-4 mutant epidermis, the basal keratinocyte progenitors that lost centrioles upregulated p53 and only a small fraction of cells died ( 5\% with CI.CASP3) (Fig. 2a-d). In newborns, the basal acentriolar keratinocytes also showed high levels of $p 53$, but only rare cells died (Fig. 2e). In contrast, centriole loss in the early embryo and developing brain led to widespread cell death and tissue degeneration ${ }^{23,24}$. The data indicate that the skin epidermal keratinocytes are more robust than early embryonic and developing brain cells, suggesting that basal keratinocytes adapt to centriole loss to maintain a relatively intact skin barrier (Fig. 1d). Our findings are consistent with the human skin phenotypes not being prominent features in patients with mutations in genes encoding centrosomal proteins ${ }^{22}$. The Café au Lait skin pigmentation defect is a rarely reported skin phenotype in these patients ${ }^{46,47}$. These skin pigmentation defects are likely due to high p53 and the tanning effect ${ }^{48}$.

The skin epithelial centrosome mutants had a thin epidermis and sparse hair (Fig. 1e-f). These two prominent centrosome-associated phenotypes were dependent on the activation of the mitotic surveillance pathway because they were rescued upon the removal of $p 53,53 \mathrm{BP} 1$ or USP28 (Fig. 3a-d). Given that p63 is the master transcription factor governing epidermal stratification and skin appendage development ${ }^{4}$, and that p53 and p63 share a consensus DNA binding site, our RNA-Seq data (Fig. 2h-j; Tables S1 and S2) suggested that the abnormal increase in p53 levels in the developing skin epithelium of centrosome mutants disrupts p63 functions leading to the skin defects. In addition, the rescue of the activation of the mitotic 
surveillance pathway using 53bp1 or Usp28 mutations in the centrosome mutant skin epithelium confirms the conservation of this pathway in mammalian tissues in vivo.

Many signaling pathways have been associated with centrosomes and their extensions, the primary cilia ${ }^{49}$. The phenotypes of the skin epithelial centrosome double mutants (Fig. 3b-d), which also lack cilia, and Ift88/cilia mutants (Fig. 1e-g), confirm earlier reports about the requirement of cilia and cilia-associated signaling mainly in postnatal hair follicles during homeostasis ${ }^{33}$. Our genetic data do not support a major link between centrosomes or cilia and Notch signaling in the skin epithelium ${ }^{34}$, where Notch signaling plays roles in epidermal and hair shaft differentiation ${ }^{6,42,50}$.

During the first phase of epidermal development between E13-E15, our data show that the daughter cells of the basal progenitors that point away from the epidermal-dermal interface in a perpendicular division can be incorporated in the basal layer following division (Fig. 5a, Movie S1). Moreover, the inhibition of cell division in the developing skin does not completely abolish cellular differentiation and stratification and may be compensated by other mechanisms (Fig. S5a-e). Indeed, our time-lapse imaging data show that cellular delamination from the basal layer is a possible mechanism of stratification (Fig. 5c; Movie S3), with published evidence supporting or excluding this mechanism in the epidermis ${ }^{18,51-54}$. The delaminating cells may act as the transit-amplifying population because they divide right after delamination in the suprabasal layers. In addition, cortical tension and cell adhesion have been shown to regulate the balance between cell proliferation and differentiation in skin epidermal keratinocytes in vitro ${ }^{53}$. The fastexpanding skin in the developing embryo is under tension and the tissue tension appears to be released in the skin explant cultures. However, the epidermis in the explants retains the ability to stratify and differentiate (Fig. S5g,h), particularly in the new skin roll cultures that are embedded in a matrix (see Materials and Methods). The skin roll explants seem to better 
recapitulate the epidermal stratification program in vivo (Fig. S5g), perhaps due to the pressure offered by the embedding matrix. The difference between the two skin explant methods suggests that stratification and differentiation may also be uncoupled. Together with the timelapse imaging data, our findings suggest that local cell-cell interactions and tension differentials, rather than global tissue tension, might dictate cell fate choices within the basal layer of the epidermis.

During the second phase of epidermal development between E15.5-E17.5, the Sas-4 p53 mutants show a decrease in the fraction of parallel division orientation and an increase in the perpendicular one in basal keratinocyte progenitors that do not lead to a corresponding decrease in basal layer density, or a suprabasal layer thickening (Fig. 4e-g), as predicted by the model that cell division orientation and epidermal differentiation are coupled ${ }^{55}$. Of note, the increase in cell densities and cell packing defects in both layers of the epidermis in Sas-4 p53 double mutants (Fig. 4g-j) seem to be dependent on p53 and/or cilia (Fig. S4c,d), and importantly, the p53 single mutants do not significantly shift their cell division orientation relative to controls at E16.5 (Fig. S4a). It is interesting to note that the proportional increase in cell densities in both layers in these mutants at E16.5 is consistent with our data suggesting an equilibrium and a counting mechanism across the epidermal layers during this stage (Fig. 6c; Fig. S6d). Collectively, our data suggest that a perpendicular division of the basal progenitors during this phase does not necessarily result in an asymmetric cell fate. It has been known for a few decades that switching primary mouse skin epidermal keratinocytes in culture to high calcium levels induces differentiation independent of cell division ${ }^{56}$. The data suggest that commitment to differentiation in the basal progenitors is a multi-step process that is independent of division orientation, and is associated with cellular detachment or delamination, similar to earlier reports in the developing and adult mouse skin epidermis as well as in human skin 
equivalents ${ }^{52-54}$. It is worth noting that perpendicular divisions have only been rarely observed in the adult skin epidermis ${ }^{52,57}$.

It has recently been shown that the cellular geometry of the epidermal basal progenitors correlates with cell division orientation in different body sites in the developing embryo at E14.5 ${ }^{58}$. However, all the skin body sites generate a stratified epidermis and a fully functional barrier by $\mathrm{E} 17.5$ (Fig. 1d) ${ }^{7}$. Our data support a model whereby a thin back-skin epidermis at E12.5 mechanically favors a parallel division orientation in basal keratinocytes. As development proceeds and more suprabasal layers are added, the thickened epidermis allows perpendicular cell division orientation in the basal layer. Then, in adult life the skin epidermis is thin again and predominantly ensures parallel division orientation of basal keratinocytes. In our opinion, the correlations between a thinner epidermis and a parallel division orientation, or between a thicker epidermis and a perpendicular division orientation of the progenitors, can be explained by the topology of the epidermis restraining the cell division orientation, rather than the prevailing opposite causal relationship ${ }^{10,59}$. One example in this study is the Sas-4 single mutant, which shows a thinner epidermis and has a higher proportion of parallel divisions (Fig. S1b).

In summary, our data support a two-phase model of physiological epidermal stratification (Fig. 7b). The first phase between E12.5-E15.5 is the main phase of stratification and is fueled by the higher proliferation rates of the basal cells and the newly-produced K10-positive, transitamplifying and suprabasally-committed cells, which originate from the basal layer by cellular delamination (Fig. 7b). The second phase between E15.5-E18.5 is a maintenance phase which is perhaps supported by cellular delamination from the basal layer, and is contiguous with postnatal epidermal growth and turnover (Fig. 7b). Why and how certain basal progenitor cells commit to a cell fate switch, delamination and differentiation in either phase are still open questions. For the first phase, cellular crowding and chance extrusion may be the dominant 

available under aCC-BY-NC-ND 4.0 International license.

forces ${ }^{53}$. Perhaps the choice to delaminate is stochastic in nature, as has been shown for the adult skin epidermis and even other epithelia ${ }^{52,57}$. In this respect, a weak noisy signal, such as Notch signaling, is amplified and fixed by the cells committed to delaminate and differentiate, while concomitantly inhibiting the surrounding cells from adopting the same fate $6,42,60,61$. 


\section{Acknowledgement}

We thank our colleagues at the University of Cologne and SFB829: Carien Niessen, Sara Wickström (University of Helsinki, Finland), Sandra Iden (Saarland University, Germany), Catherin Niemann, Mirka Uhlirova and Leo Kurian for critical discussions about the work and comments on the manuscript. We thank Terry Lechler (Duke University, USA) and Scott Williams (University of North Carolina, USA) for valuable suggestions during their sabbaticals in Cologne. We thank David Gonzalez and Valentina Greco (Yale University, USA) for their advice on time-lapse imaging analyses. We acknowledge and appreciate the CECAD in vivo research facility (Branko Zevnik) for the generation of the new mouse lines of 53bp1 and Usp28, and rederivation and maintenance of the other lines. We thank the CECAD imaging facility, especially Peter Zentis for image analyses, and Hans Fried and Christoph Möhl of the DZNE Imaging and Data Analysis Facilities (Bonn, Germany). Special thanks to Hironobu Fujiwara and Ritsuko Morita (RIKEN Center, Japan) for sharing unpublished data on vibrissae pad imaging. We acknowledge the SFB829 Z4 platform (Cornelia Mauch and Doris Helbig) for providing paraffin sections of normal and hyperproliferative human skin samples. The work was funded by the Deutsche Forschungsgemeinschaft (DFG, German Research Foundation)- Project-ID 73111208 - SFB829 “Molecular Mechanisms regulating Skin Homeostasis”. The project was also supported by the DFG under Germany's Excellence Strategy - CECAD, EXC 2030 390661388. The funders had no role in study design, data collection and analysis, decision to publish, or preparation of the manuscript.

\section{Author contributions}

Conceptualization: H.B. and M.D.; Methodology: H.B., M.D., L.W. and B.D.S.; Software: E.S., M.D., L.W. and H.B.; Formal Analysis: M.D, L.W., H.B., E.S. and B.D.S.; Investigation: M.D., L.W., E.S., H.K. and H.B.; Resources: C.K.; Writing: H.B., M.D. and L.W.; Visualization: M.D., L.W. and H.B.; Supervision, Project administration and Funding Acquisition: H.B. 
bioRxiv preprint doi: https://doi.org/10.1101/2020.07.24.219493; this version posted January 20, 2021. The copyright holder for this preprint (which was not certified by peer review) is the author/funder, who has granted bioRxiv a license to display the preprint in perpetuity. It is made available under aCC-BY-NC-ND 4.0 International license.

\section{Declaration of interests}

The authors declare no competing interests. 


\section{Methods}

\section{Animals and Genotyping}

The following mouse alleles were used in this study: Sas- $4^{\mathrm{fff}}$ (Cenpj $\left.{ }^{\mathrm{tm} 1 \mathrm{C}(\mathrm{EUCOMM}) \mathrm{Wtsi} / \mathrm{tm} 1 \mathrm{~d}(\mathrm{EUCOMM}) \text { Wtsi }}\right)$

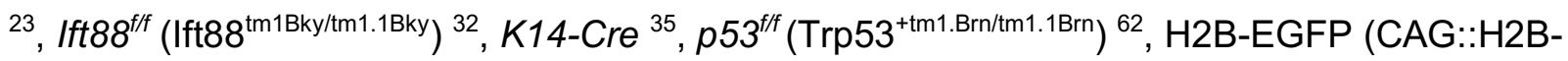
EGFP) ${ }^{63}$. The CRISPR/Cas9 endonuclease-mediated knockout (em) mouse knockouts of $53 b p 1^{e m / e m}\left(\operatorname{Trp53bp} 1^{\mathrm{em} / \mathrm{em}}\right)$ and Usp28 $8^{\mathrm{em} / \mathrm{em}}$ were generated by the CECAD in vivo Research Facility (ivRF) using microinjection of the corresponding gRNA, Cas9 mRNA and Cas9 protein into fertilized zygotes:

\begin{tabular}{|l|l|l|}
\hline & $\mathbf{5 3 b p 1}$ & Usp28 \\
\hline Exon & Exon 2 & Exon 2 \\
\hline gRNA & TACTGGAAGTCAATTGGATT & AATCAGCTGCGAGAAATCAC \\
\hline Mutation & 31 bp deletion & 16 bp deletion \\
\hline Deleted & GGACCCTACTGGAAGTCAATTGGATTCAGAT & CTGCGAGAAATCACAG \\
\hline Primer 1 & TCGAACTGATCTTTTGTTATTCCA & TCAAAAACAGAGCTGCCAGA \\
\hline Primer $\mathbf{2}$ & GAACAGGGCATCATCACTCA & CACACCTGACATGTGGGAAA \\
\hline
\end{tabular}

All phenotypes were analyzed in the FVB/NRj background. The littermates that had the Crerecombinase and were heterozygous for the floxed or knockout alleles were used as preferred controls where available. Genotyping was carried out using published or standard PCR protocols.

The animals were generated, housed and bred under standard conditions in the CECAD ivRF. The generation and breeding described were approved by the Landesamt für Natur, Umwelt, und Verbraucherschutz Nordrhein-Westfalen (LANUV), Germany (animal applications: 8402.04.2014.A372, 84-02.04.2015.A405 and 81-02.04.2019.A476). 


\section{Histological analysis}

Skin or embryo samples were fixed overnight in $10 \%$ Formalin or $4 \%$ Paraformaldehyde (PFA), respectively, washed with $1 x$ PBS and stored in $70 \%$ ethanol for several days. After dehydration, the samples were embedded in paraffin and sectioned on an RM2255 microtome (Leica Biosystems) at $8 \mu \mathrm{m}$. For histological analyses, the skin sections were stained with hematoxylin and eosin (H\&E) and mounted with Entellan® (Merck). The stained slides were imaged using a DM2000 light microscope (Leica biosystems) or scanned with an SCN400 Slide scanner (Leica biosystems) for subsequent analyses.

\section{Immunofluorescence and Imaging}

Embryos were fixed in $4 \%$ PFA overnight at $4{ }^{\circ} \mathrm{C}$, washed with $1 \times$ PBS and then cryoprotected in $10-30 \%$ sucrose in $1 \times$ PBS overnight at $4{ }^{\circ} \mathrm{C}$. After embedding in Tissue-Tek® (Optimal Cutting Temperature Compound (OCT), Sakura Finetek USA INC), the blocks were sectioned on a CM1850 Cryostat (Leica Biosystems) at 7-10 $\mu \mathrm{m}$. Postnatal skin samples for immunofluorescence staining were fresh-frozen in OCT and sectioned as above.

For immunofluorescence staining, skin sections were fixed for 10 minutes (min) in $4 \%$ PFA and washed with 1x PBS prior to the common staining protocol: skin or embryo sections were fixed in ice-cold methanol for $10 \mathrm{~min}$ at $-20{ }^{\circ} \mathrm{C}$, washed with washing buffer containing $0.2 \% \operatorname{Triton}^{\mathrm{TM}}$ $\mathrm{X}-100$ in $1 \mathrm{x}$ PBS and blocked for 1 hour $(\mathrm{h})$ in blocking buffer containing washing buffer and heat-inactivated goat serum ( $1 \%$ for embryo and $10 \%$ for skin sections). Mouse IgG Fab fragments were used at 1:10 to block background staining when using mouse primary antibodies (cat\#115-007-003, Jackson Laboratories). After the primary antibodies were incubated over night at $4{ }^{\circ} \mathrm{C}$, the sections were washed with washing buffer, incubated with the secondary antibody and DAPI for $1 \mathrm{~h}$ at RT and mounted with Prolong Gold (Cell Signaling). Images were obtained using a SP8 confocal microscope (Leica microsystems) or a Meta 710 confocal microscope (Zeiss). 
For p63, p73 and p21 immunostaining, paraffin-embedded embryos were sectioned at $5 \mu \mathrm{m}$.

Antigen retrieval of deparaffinized sections was performed using Retrieve-All buffer

(BioLegend). Paraffin sections of human skin samples were obtained from the SFB829 Z4

biobank, deparaffinized and rehydrated before performing antigen retrieval by boiling in citrate buffer followed by the common staining protocol described above.

\section{Antibodies}

The following primary antibodies were used in this study:

\begin{tabular}{|l|l|l|l|l|}
\hline Antigen & $\begin{array}{l}\text { Source/ } \\
\text { Isotype }\end{array}$ & Company & $\begin{array}{l}\text { Catalog } \\
\text { number }\end{array}$ & $\begin{array}{l}\text { Working } \\
\text { dilution }\end{array}$ \\
\hline 53BP1 & rabbit & Novus Biologicals & NB100-305SS & $1: 1000$ \\
\hline ARL13B & rabbit & Proteintech & $17711-1-A P$ & $1: 1000$ \\
\hline CEP164 & rabbit & Proteintech & $22227-1-A P$ & $1: 2000$ \\
\hline Cleaved-Caspase3 & rabbit & Cell Signaling & 9661 & $1: 400$ \\
\hline Collagen type IV & rabbit & Merck & AB756P & $1: 400$ \\
\hline Integrin-a6 & rat IgG2a & R\&D Systems & MAB13501 & $1: 1000$ \\
\hline Keratin1 & rabbit & BioLegend & 905601 & $1: 4000$ \\
\hline Keratin10 & guinea pig & Progen & GP-K10 & $1: 400$ \\
\hline Keratin14 & guinea pig & Progen & GP-CK14 & $1: 400$ \\
\hline Keratin6A & rabbit & BioLegend & 905701 & $1: 4000$ \\
\hline Ki67 & rabbit & ThermoFisher & PA5-19462 & $1: 400$ \\
\hline Laminin & rabbit & Sigma Aldrich & L9393 & $1: 1000$ \\
\hline Loricrin & rabbit & BioLegend & 905101 & $1: 2000$ \\
\hline p21 & mouse & Santa Cruz & sc-6246 & $1: 50$ \\
\hline p53 (CM5) & rabbit & Linaris & LIN-P956 & $1: 1000$ \\
\hline p63 & mouse & Abcam & ab735 & $1: 25$ \\
\hline
\end{tabular}




\begin{tabular}{|l|l|l|l|l|}
\hline p73 & rabbit & Abcam & ab40658 & $1: 1000$ \\
\hline Phospho-Histone H3 & rabbit & Merck & $06-570$ & $1: 400$ \\
\hline Survivin & rabbit & Cell Signaling & 2808 & $1: 400$ \\
\hline USP28 & rabbit & Sigma Aldrich & HPA006778 & $1: 500$ \\
\hline Y-Tubulin & mouse IgG1 & Sigma Aldrich & T6557 & $1: 2000$ \\
\hline
\end{tabular}

Secondary antibodies were Alexafluor ${ }^{\circledR} 488,568$, or 647 conjugates (Life Technologies, Invitrogen (ThermoFisher)) and used at 1:1000 in combination with DAPI at 1:1000 (AppliChem A4099).

\section{Keratinocyte isolation}

Newborn mice were decapitated, transferred through a disinfection series (Betaisodona, 1x PBS 1:1; Octenisept; 1x PBS; $70 \%$ ethanol; 1x PBS; antibiotic/antimycotic solution in 1x PBS 1:100) and skinned. The skins were incubated overnight at $4{ }^{\circ} \mathrm{C}$ in a $5 \mathrm{mg} / \mathrm{ml}$ dispase II solution in DMEM/Hams-F12 (without supplements). After the epidermis was separated from the dermis, the epidermis was floated with the basal side down on $1 \mathrm{ml}$ of $0.25 \%$ Trypsin (without EDTA, Gibco) for 20 min at room temperature (RT). Then, keratinocyte medium was used to dissociate the keratinocytes from the epidermis ${ }^{56,64}$.

\section{RNA isolation and RNA-Seq analyses}

RNA was prepared from frozen keratinocytes, which were isolated as described above without plating, or from frozen epidermal sheets of E13.5 embryos. Embryonic skin at E13.5 was microdissected and enzymatically separated into epidermis and dermis as described previously ${ }^{65}$. Briefly, the skin was incubated with 2:1 EDTA-free trypsin/pancreatin solution for 5 min at RT and 30 min on ice before separating the epidermis. Total RNA was isolated using the RNeasy Plus Mini Kit (Qiagen). RNA samples were submitted to the Cologne Center for Genomics 
(CCG), where quality check and RNA-Seq were performed. The raw data were processed by the CECAD Bioinformatics facility using the QuickNGS pipeline to generate differentially expressed genes (fold change $\geq 2$, $p$-value $\leq 0.05)^{66}$. For more information on the RNA-Seq procedure, please check the GEO deposited metadata file. Overlap of differentially expressed genes was determined and visualized in Venn diagrams using BioVenn ${ }^{67}$. Comparison of differentially expressed genes with canonical pathways gene sets derived from the PID pathway database ${ }^{68}$ was analyzed using the Molecular Signatures Database v7.2 of GSEA ${ }^{69}$.

\section{Skin barrier assay}

E17.5 or E18.5 embryos were sacrificed by making a cut in the neck to sever the spinal cord, and tail tips were taken for genotyping. After incubating the embryos for 2 min each in an increasing and then decreasing methanol series and washing in $1 \mathrm{x}$ PBS, they were stained in a $0.1 \%$ toluidine blue solution in water for 1-2 min on ice. A specific dye pattern showing possible barrier defects appeared after de-staining the embryos in 1x PBS on ice ${ }^{7,70}$.

\section{Cell cycle analysis}

Freshly isolated epidermal keratinocytes were fixed in $70 \%$ ethanol and stored at $-20{ }^{\circ} \mathrm{C}$ for several weeks. Then they were centrifuged, washed in 1x PBS and resuspended in Propidium lodide staining solution (10 $\mu \mathrm{g} / \mathrm{ml} \mathrm{PI}, 200 \mu \mathrm{g} / \mathrm{ml}$ RNAse A, $0.1 \% \operatorname{Triton}^{\mathrm{TM}} \mathrm{X}-100$ in $1 \mathrm{x}$ PBS). After incubating at RT for 30 mins, cell cycle analysis was performed using a LSRFortessa (BD) FACS machine. Data were analyzed using the FlowJo software.

\section{Skin explant culture and EdU assays}

Lateral skin explants were taken from E13.5 wild-type embryos and placed on Nucleopore Track-Etch membranes (Whatman) floating on DMEM (Gibco) containing $10 \%$ FBS and 1x antibiotic/antimycotic solution (Gibco) at $37^{\circ} \mathrm{C}$ and $5 \% \mathrm{CO}_{2}$. The explants were treated with 
either $10 \mu \mathrm{g} / \mathrm{ml}$ Mitomycin C or DMSO (vehicle) in medium for $3 \mathrm{~h}$, washed with 1x PBS and cultured further in the medium above. Either directly at E13.5, after 1 day (E14.5) or after 2 days (E15.5), $20 \mu \mathrm{M} \mathrm{EdU}$ was added to the media for $2 \mathrm{~h}$, then the explants were washed with $1 \mathrm{x}$ PBS and fixed in $4 \%$ PFA for $2 \mathrm{~h}$ at RT. After several washes with $1 \mathrm{x}$ PBS, the explants were removed from the membrane, cryoprotected in $30 \%$ sucrose overnight at $4{ }^{\circ} \mathrm{C}$ and embedded in OCT for immunofluorescence staining (same protocol as for whole embryo sections except using $0.1 \%$ Triton $^{\mathrm{TM}} \mathrm{X}-100$ in PBS). For EdU analyses in embryonic mouse skin, the pregnant females at the corresponding timepoints were injected intra-peritoneally with EdU at $50 \mathrm{mg} / \mathrm{kg}$ and then anesthetized and sacrificed 3h later for embryo collection, fixation and OCT embedding as described above. The skin sections were treated according to the Click-iT ${ }^{\mathrm{TM}} \mathrm{EdU}$ imaging kit (Thermo Fisher) instructions before performing regular immunofluorescence staining.

\section{Time-lapse Imaging}

Lateral skin explants were taken from E13.5 embryos (H2B-EGFP ${ }^{\mathrm{tg} / \mathrm{wt}}$ ) and transferred to media (advanced DMEM + $2 \mathrm{mM} \mathrm{L-Glutamine}+0.1 \mathrm{mg} / \mathrm{ml}$ Penicillin/Streptomycin $+10 \%$ FBS) at $37^{\circ} \mathrm{C}$ where they formed rolls. The rolls were embedded in $1 \%$ low-melting agarose or Matrigel (Corning) in media at $37^{\circ} \mathrm{C}$, where the cutting edge was touching the membrane-bottom of a Lumox $®$ dish (Sarstedt) or a glass-bottom dish (ibidi). The dishes were covered with media and incubated at $37^{\circ} \mathrm{C}$ and $5 \% \mathrm{CO}_{2}$ until imaging. Time-lapse imaging was carried out between E13.5 and E14.5 using an inverted SP8 confocal microscope (Leica microsystems), an inverted Dragonfly Spinning disc confocal microscope (Andor) or an inverted LSM710NLO Two-Photon microscope (Zeiss) with 20x air objective or 40x water or oil immersion objective and incubation at $37^{\circ} \mathrm{C}$ and $5 \% \mathrm{CO}_{2}$. 


\section{Image analyses}

Epidermal thickness and number of hair follicles were quantified by scanning the histological sections with the SCN400 slide scanner (Leica Biosystems) and analyzing the images using the Digital Image Hub (Leica Biosystems). The percentage of p53-positive cells in the epidermis was obtained using ImageJ (NIH) and CellProfiler (Broad institute). The clusters of CI.CASP3positive cells, the thickness of $\mathrm{K} 1$ or $\mathrm{K} 10$ layers, the number and average size of basal and suprabasal layer cells, the Ki67-positive cells, the pHH3-positive cells as well as the centrosome- or cilia-containing cells in the epidermis were analyzed using ImageJ. The angles of basal cell division orientation were obtained from late anaphase or telophase cells in the epidermis by measuring the angle of the division axis (marked by Survivin) with the basement membrane (ITGA6) using ImageJ. The radial histograms were plotted using the OriginPro® software (Origin Lab), while all the other diagrams were generated using Prism (GraphPad). Time-lapse imaging data were manually analyzed using ImageJ (Correct 3D Drift plug-in), Volocity (Improvision) and Imaris (Bitplane).

\section{Statistical Analysis}

Two groups or more of data were compared using a two-tailed student's T-test with a cutoff for significance of $<0.05$, or one-way ANOVA and Tukey's multiple comparisons test, both of which gave similar significance outcomes (Excel or GraphPad Prism). Angle measurements were compared using a two-way ANOVA, Kolmogorov-Smirnov Test and Chi squared Test (GraphPad Prism). The data are presented as the mean \pm SD (standard deviation).

\section{Supplementary Theory}

In the following, we consider the basis of the "two-phase" model of embryonic epidermal development based on measurements of the net growth of the embryo, the change in the basal and superbasal cell density, and estimates of the cell proliferation rate within the two layers. To 
determine the overall increase in epidermal basal and suprabasal cell numbers during development, we must combine estimates of the net increase in tissue area (as inferred from the net expansion of the embryo) and cell density. Both are measured with respect to anteroposterior (sagittal) and dorsoventral (transverse, hereafter called orthogonal) axes of the mouse (Fig. 6a; Fig. S6b). Notably, the length of the of the embryo along both axes, $l_{\mathrm{s}}(t)$ and $l_{\mathrm{o}}(t)$, grows approximately linear with time over the entire E12.5-E18.5 time-course (Fig. 6a; Fig. S6b). Alongside this increase, there is also a change in the basal and suprabasal cell density, with the data showing a small differential between the sagittal and orthogonal directions, most likely due to the differential expansion rates along the body axes (Fig. 6b,c; Fig. S6c,d). Therefore, if we define $\rho_{\mathrm{b} / \mathrm{s}, \mathrm{s} / \mathrm{o}}$ as the basal/suprabasal cell density in the sagittal/orthogonal directions, the total basal/suprabasal cell numbers increase in proportion to

$$
n_{\mathrm{b} / \mathrm{s}}(t)=\rho_{\mathrm{b} / \mathrm{s}, \mathrm{s}} l_{\mathrm{s}} \times \rho_{\mathrm{b} / \mathrm{s}, \mathrm{o}} l_{\mathrm{o}}
$$

Based on this estimate, over the first three days from E12.5 to E15.5, the total cell numbers (basal and suprabasal) increase approximately exponentially with time, rising by a factor of around 7 (Fig. 6c,e; Fig. S6d). Notably, this coincides with the time period in which proliferative cells are found in both basal and suprabasal cell layers (Fig. 6d; Fig. S6e). After this period, the cell number increase in both basal and suprabasal cell layers is greatly reduced, showing only a factor of 2 increase over the next three days (Fig. 6c,e; Fig. S6d).

Together, these results suggest a "two-phase" behavior, with an early phase of cell amplification through rounds of cell duplication, followed by a second phase in which basal cells steadily expand to meet the demands of the underlying growing tissue while, at the same time, giving rise to non-cycling suprabasal cells at a rate that allows both layers to expand in an approximately proportionate manner (Fig. 6c; Fig. S6d). Significantly, during the early phase of 
cell amplification, cells delaminate from the basal layer (potentially by the effects of cell crowding), giving rise to a proliferatively active suprabasal cell layer.

Therefore, to capture quantitatively the first phase of cell amplification, we suppose that the skin epidermis is comprised of equipotent progenitor cells, $p$, that duplicate through division at a constant rate $\lambda=0.66$ per day.

$$
p \stackrel{\lambda}{\rightarrow} p+p
$$

Initially (E12.5-E13.5), this increase in cell number is accommodated through the ongoing expansion of the basal cell layer. During the next day (E13.5-E14.5), the basal cell density becomes elevated slightly, while an excess of proliferative cells is transferred - potentially through crowding - to the suprabasal cell layer. This trend continues during the third day of study (E14.5-E15.5), after which the ratio of basal to suprabasal cells reaches roughly parity (Fig. 6c; Fig. S6d). At this time point, the frequency of proliferative suprabasal cells diminishes significantly (Fig. 6d; Fig. S6e), suggesting that this last phase is characterized by cell cycle exit consistent with the cells' entry into a terminal differentiation program.

After this point, the data suggest only a modest rate of increase in the number of basal and suprabasal cells, which rise proportionately by a factor of around 2 over the next three days from E15.5 to E18.5 (Fig. 6c,e; Fig. S6d). Although the experimental data is noisy, the measured increase has a linear-like trend. To model the dynamics in this phase (Fig. 6e), we suppose that the basal cell layer is comprised of a single equipotent progenitor cell population, $b$, and a single non-proliferative suprabasal cell population, $s$, defined by the kinetics ${ }^{52}$,

$$
b \stackrel{\lambda}{\rightarrow}\left(\begin{array}{cc}
b+b & \text { Pr. } 1-r \\
s & \text { Pr. } r
\end{array}\right.
$$


so that at an overall rate $\lambda$, with probability $1-r$, basal cells duplicate, while with probability $r$ they commit to terminal differentiation and stratify into the suprabasal layer, i.e.

$$
\dot{b}=\lambda(1-2 r) b, \quad \dot{s}=\lambda r b
$$

To ensure that suprabasal cells are produced in proportion to basal cells, $\dot{b}=\dot{s}$, i.e. $r=1 / 3$. Further, if we propose that, over the three-day time course, the proliferation rate, $\lambda$, is approximately constant, we have that

$$
b=b_{0} e^{\lambda\left(t-t_{0}\right) / 3}=s
$$

where $b_{0}$ denotes the basal cell number at time $t_{0}=15.5$ days. With basal cell number increasing by approximately 2 over the three-day time course, this translates to a rate $\lambda=0.7$ per day, similar to the estimated division rate during the early amplification phase.

As a consistency check, we can question what would be the expected EdU incorporation rate in the basal cell layer. With an S-phase of around $t_{s}=6-8$ hours, and a cell cycle time $1 / \lambda$, we would expect a short-term EdU pulse to mark a fraction of $\lambda t_{s} \sim 0.8 \times(1 / 3)=0.27$ of cells during the early phase. For a longer pulse ( 3-4 hours), marked cells would have progressed through one round of division, leading to a doubling of the number to around $50 \%$. This Fig. is comparable to the measured estimates from the experimental data. In the later phase, the cell division rate drops by around $40 \%$, broadly consistent with the EdU measurements (Fig. 6d).

\section{Data Availability}

The RNA-Seq data described in this study were deposited at the GEO repository under GSE161387. Source data are provided with this paper. 


\section{Figure Legends}

\section{Figure 1. Centrioles, not cilia, are important for proper epidermal and hair follicle}

formation. (a) Immunostaining of Control and K14-Cre+; Sas-4/f or K14-Cre+; Ift88 ${ }^{f / f}$ back-skin sections at E15.5 showing the loss of centrosome and centriole markers (TUGB and CEP164) or cilia marker (ARL13B). Insets are magnifications of the indicated dotted areas in each panel. Dashed line represents the epidermal-dermal interface in all panels (scale bar: $10 \mu \mathrm{m}$; inset: 3 $\mu \mathrm{m})$. (b) Quantification of the centrosome- or cilia-containing cells in (a). ${ }^{* *} p<0.01,{ }^{* * *} p<$ 0.0001 (two-tailed student's T-test). Bars represent mean \pm SD (standard deviation). (c) Schematic of the epidermis depicting the different epidermal layers. (d) Toluidine Blue dyepenetration assay of Control and $\mathrm{K} 14-\mathrm{Cre}+$; Sas $-4^{\mathrm{f} / \mathrm{f}}$ embryos at E17.5 and E18.5. Arrowheads indicate regions with delayed barrier formation in $\mathrm{K} 14-\mathrm{Cre}+$; Sas $-4^{\mathrm{f} / f}$ embryos (scale bar: $2 \mathrm{~mm}$ ). (e) Gross phenotypes of Control, K14-Cre+; Sas- $4^{f / f}$ and K14-Cre+; Ift88 $8^{f / f}$ mice are shown at P21 (scale bar: $1 \mathrm{~cm}$ ), as well as H\&E histological staining of back-skin sections at P0 (scale bar: $100 \mu \mathrm{m}) .(\mathbf{f}, \mathbf{g})$ Quantification of the interfollicular epidermal thickness $(\mathbf{f})$, excluding the cornified layer, or the number of hair follicles $(\mathbf{g})$ at P0 of Control, K14-Cre+; Sas- $4^{\mathrm{f} / f}$ and K14Cre+; Ift88 ${ }^{f / f}$ mice. ${ }^{* * *} \mathrm{p}<0.0001$ (two-tailed student's T-test or one-way ANOVA and Tukey's multiple comparisons test). Bars represent mean \pm SD. (h) Immunostaining of Control, K14Cre+; Sas-4 $4^{f / f}$ and $\mathrm{K} 14-\mathrm{Cre}+;$ Ift88 ${ }^{\mathrm{fff}}$ back-skin sections at P0 stained for the epidermal layers' markers $\mathrm{K} 14, \mathrm{~K} 1$ and Loricrin (LOR), the activation marker $\mathrm{K} 6 \mathrm{~A}$ and the basement membrane marker Integrin-a6 (ITGA6) (scale bar: $50 \mu \mathrm{m}$ ).

Figure 2. Upregulation of p53 in the centrosome mutant epidermis. (a) Immunostaining of Control and K14-Cre+; Sas-4/f back-skin sections at the indicated stages showing the loss of the centrosome marker (TUBG) and gradual increase in nuclear p53 in the mutant mice (scale bar: $20 \mu \mathrm{m})$. Dashed line represents the epidermal-dermal interface in all panels. (b) Quantification of the percentage of p53-positive nuclei in the back-skin basal epidermis of 
Control and K14-Cre+; Sas- $4^{f / f}$ mice at the corresponding stage shown in (a). Bars represent mean \pm SD in these and subsequent graphs. $(\mathbf{c}, \mathbf{d})$ Immunostaining (c) and quantification (d) of Control and K14-Cre+; Sas-4/f back-skin sections at E15.5 for cell death (Cl.CASP3) in the mutant epidermis (scale bar: $20 \mu \mathrm{m}$ ). (e) Similar to (d) but at P0. (f) Cell cycle profiles of isolated primary keratinocytes at P0 of Control and K14-Cre+; Sas-4 $4^{f / f}$ mice. (g) Quantification of the percentage of $\mathrm{pHH} 3$-positive cells in the basal layer of back-skin epidermal sections at E15.5 of Control, K14-Cre+; Sas-4 ${ }^{f / f}$, K14-Cre+; Sas-4 $4^{f / w} ; p 53^{f / f}$ and K14-Cre+; Sas-4 $4^{f / f} ; p 53^{f / f}$ mice. n. s. = not significant, ${ }^{*} p<0.05$ (two-tailed student's T-test). (h) Bar chart depicting the over-represented pathways (from the pathway interaction database (PID)) of differentially expressed genes in the $\mathrm{K} 14-\mathrm{Cre}+$; Sas $-4^{f / f}$ epidermis compared to controls at E13.5. The X-axis represents the negative $\log _{10}$ of the q-values, the adjusted $p$-values optimized by the false discovery rate. (i) Similar to (h) but for P0 epidermal keratinocytes. (j) Venn diagram showing the overlap of published p63 target genes ${ }^{37}$ with differentially expressed genes of $\mathrm{K} 14-\mathrm{Cre}+$; Sas $-4^{f / f}$ at E13.5 and P0.

Figure 3. The skin epidermal phenotypes in centrosome mutants are due to the activation of the mitotic surveillance pathway. (a) Centrioles, besides providing the essential template for cilia, ensure efficient mitosis and repress the activation of the mitotic surveillance pathway: p53 stabilization mediated by 53BP1 and USP28 leading to cell death (mainly shown in vivo) or cell cycle arrest (mainly shown in vitro). (b) Gross phenotypes of Control, K14-Cre+; Sas-4 ${ }^{f / f}$,

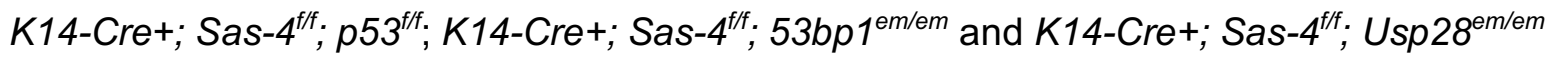
mice are shown at P21 (scale bar: $1 \mathrm{~cm}$ ), as well as H\&E histological staining of back-skin sections at P0 (scale bar: $100 \mu \mathrm{m})$. (c,d) Quantification of the interfollicular epidermal thickness (c), excluding the cornified layer, or the number of hair follicles (d) at P0 of the mouse genotypes shown in (b). Control and $\mathrm{K} 14-\mathrm{Cre}+$; Sas $-4^{\mathrm{f} / f}$ animals are part of the same experiment in Fig. 1. ${ }^{* * *} p<0.001,{ }^{* * * *} p<0.0001$ (two-tailed student's T-test or one-way 
ANOVA and Tukey's multiple comparisons test). Bars represent mean \pm SD. (e)

Immunostaining of Control, K14-Cre+; Sas-4 ${ }^{f / f}$ and K14-Cre+; Sas-4 ${ }^{f / f} ; p 53^{f / f}$ back-skin sections at P0 for K6A and ITGA6 (scale bar: $25 \mu \mathrm{m}$ ).

Figure 4. The proportions of cell division orientation in the basal progenitors do not correlate with differentiation at late stages in Sas-4 p53 mutants. (a) Representative images of parallel and perpendicular dividing basal cells from immunostaining (Survivin and ITGA6) of back-skin sections of the interfollicular epidermis of Controls at E16.5 (scale bar: 10 $\mu \mathrm{m})$. (b) Radial histograms of the distribution of the angles of cell division orientation in late anaphase to telophase of basal epidermal cells of Controls and K14-Cre+; Sas-4fff; $p 53^{f / f}$ animals at E15.5. Percentages of parallel $\left(0-29^{\circ}\right)$, oblique $\left(30-59^{\circ}\right)$ and perpendicular $(60-$ $90^{\circ}$ ) dividing basal layer cells of Controls and K14-Cre+; Sas $-4^{f / f} ; p 53^{f / f}$ animals at E15.5. (c) Similar to (b) at E16.5. (d) Similar to (b) at E17.5. (e,f) Immunostaining (e) and quantification (f) of of the differentiated (K1-positive) layer in back-skin sections at E16.5 of Control and K14Cre+; Sas $-4^{\mathrm{f} / f} ; p 53^{\mathrm{ff}}$ mice (scale bar: $50 \mu \mathrm{m}$ ). Dashed line represents the epidermal-dermal interface in all panels. (g,h) Quantification of the density (cells per $100 \mu \mathrm{m}$ ) of basal $(\mathbf{g})$ and suprabsal (h) layer cells of sagittal back-skin epidermal sections of Controls and K14-Cre+; Sas- $4^{f / f} ; p 53^{f / f}$ animals at E16.5. (i,j) Quantification of the average cell size of basal (i) and suprabasal (j) layer cells from (g) and (h), respectively. n. s. = not significant, ${ }^{*} p<0.05,{ }^{* *} p<$ 0.01 (two-tailed student's T-test). Angle measurements were compared using a two-way ANOVA, Kolmogorov-Smirnov Test and Chi squared Test, all of which gave similar statistical significance outcomes. Bars represent mean \pm SD.

Figure 5. The skin epidermal basal layer is more flexible during development. (a-c) Timelapse images of the CAG::H2B-EGFP nuclear reporter in E13.5-E14.5 skin-roll explants taken every 7 minutes. (a,b) Examples of perpendicular dividing basal cells with daughter cells that 
point away from the epidermal-dermal interface that were either integrated into the basal layer after division (a, 44 out of 94 cells), or remained in the second layer for the duration of the analysis (b, 50 out of 94 cells). (c) An Example of a delaminating cell that left the basal layer, moved suprabasally and divided (a total of 46 cells observed). Note the other dividing suprabasal cell (red asterisks) (scale bars: $10 \mu \mathrm{m}$ ). The data were obtained from 5 explants taken from 5 different embryos in 5 independent imaging experiments. Dashed line represents the epidermal-dermal interface in all panels. Yellow dots mark the cells of interest. (d) Immunostaining of back-skin sections at the indicated stages stained for ITGA6 and K10 (scale bar: $25 \mu \mathrm{m})$. Arrowheads indicate cells in the "second" layer that are positive for ITGA6 but negative for $\mathrm{K} 10$.

Figure 6. Skin epidermal stratification occurs in two phases. (a) Quantification of the crownrump distance as depicted in the embryo schematic (red line) in wild-type embryos from E12.5E18.5. Note the linear trend of the growth. $(\mathbf{b}, \mathbf{c})$ Cell densities (cells per $100 \mu \mathrm{m}$ ) of basal and suprabasal layers of sagittal back-skin sections of the epidermis from E12.5-E18.5. (c) The suprabasal cell density reaches parity with that of the basal layer starting at E15.5. (d) Quantification of EdU-positive cells in the basal and suprabasal layers from sagittal back-skin sections of the epidermis at the indicated timepoints, with an example of the EdU/K10 staining shown at E14.5 (arrowheads) (scale bar: $10 \mu \mathrm{m})$. (e) The graph depicts the exponential increase in total cell number in the first phase of stratification between E12.5-E15.5 and the linear-trend increase in the second phase between E15.5-E18.5, shown as the calculated total cell number from the measurements (total) and in agreement with the model (red line). ${ }^{*} p<$ $0.05,{ }^{* *} p<0.01,{ }^{* * *} p<0.001,{ }^{* * *} p<0.0001$ (two-tailed student's T-test). Bars represent mean $\pm S D$.

\section{Figure 7. The human epidermis harbors a transit-amplifying population and a proposed}


model of mouse skin epidermal development. (a) Immunostaining of Control, psoriasis (PSO) and atopic dermatitis (AD) human skin samples for Ki67 and K10 (scale bar: $50 \mu \mathrm{m}$ ), and quantification of the percentage of Ki67-positive cells in the basal and suprabasal layers. ${ }^{*} p<$ $0.05,{ }^{* *} p<0.01,{ }^{* * * *} p<0.0001$ (two-tailed student's T-test). Bars represent mean \pm SD. (b) An illustration of the model for mouse skin epidermal development. The simple-like epithelium between E9.5-E12.5 undergoes its main stratification events during the first phase between E13.5-E15.5, where both basal and suprabasal keratinocytes have high proliferation/division rates and basal cells delaminate to fuel stratification. In the second phase between E15.5E18.5, proliferation rates are slower, decreasing dramatically in the suprabasal layer, delamination may be a dominant mechanism in maintaining stratification during tissue growth, and the angle of cell division orientation in the basal keratinocytes may be uncoupled from epidermal differentiation. 


\section{Supplementary Figure, Movie and Table Legends}

Figure S1. Body weight and hair follicle defects upon centriole loss in stratified epithelia including the skin epidermis. (a) Measurements of the body weight of Control and K14-Cre+; Sas -4 fff mice from P0 to P20. ${ }^{*} p<0.05,{ }^{* *} p<0.01,{ }^{* * *} p<0.001$ (two-tailed student's T-test). Bars represent mean \pm SD. $(\mathbf{b}, \mathbf{c}) \mathrm{H} \& \mathrm{E}$ histological staining of back-skin sections at P8 (b) and P21 (c) of Control, K14-Cre+; Sas-4 ${ }^{f / f}$ and K14-Cre+; Ift88 $8^{f / f}$ mice (scale bars: $100 \mu \mathrm{m}$ ).

Figure S2. p53 pathway in cilia and centrosome mutant epidermis. (a) Immunostaining of Control and $\mathrm{K} 14-\mathrm{Cre}+; \mathrm{ft} 88^{\mathrm{ff}}$ back-skin sections at E15.5 showing no detectable p53 upregulation in cilia mutants (scale bar: $20 \mu \mathrm{m}$ ). TUBG marks the centrosomes. Dashed line represents the epidermal-dermal interface in all panels. (b,c) Immunostaining of p21 and p53 (b) or p63 and p73 (c) of Control and K14-Cre+; Sas-4 ${ }^{f / f}$ back-skin sections at P0 (scale bars: 80 $\mu \mathrm{m}(\mathbf{b}), 50 \mu \mathrm{m}(\mathbf{c}))$.

Figure S3. The CRISPR-Cas9-generated hypomorphic alleles for 53bp1 and Usp28 and their effect on p53 levels in centrosome mutants. (a,b) Immunostaining of back-skin sections

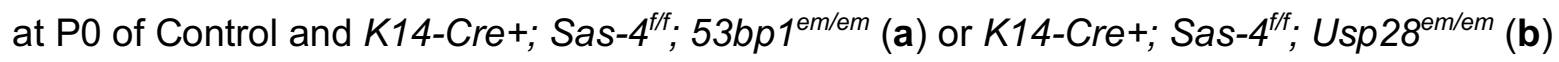
showing residual signal of 53BP1 (a) or USP28 (b) suggesting that they are hypomorphic alleles (scale bars: $50 \mu \mathrm{m})$. (c,d) Immunostaining (a) and quantification (b) of p53 in back-skin sections

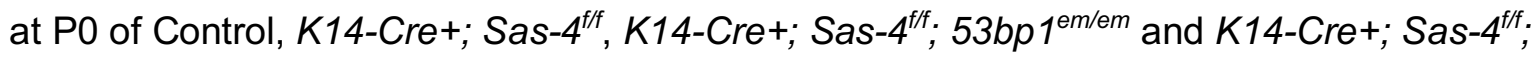
Usp28 $8^{\mathrm{em} / \mathrm{em}}$ (scale bar: $50 \mu \mathrm{m}$ ). The Control and $\mathrm{K} 14-\mathrm{Cre}+$; Sas $-4^{\mathrm{f} / f}$ are part of the same experiment in Figure $2 b .{ }^{*} p<0.05,{ }^{* * * *} p<0.0001$ (two-tailed student's T-test). Bars represent mean \pm SD.

Figure S4. Cell division orientation measurements, cell density and cell size in the epidermis of the different mutants and controls. (a,b) Radial histogram distribution of the 
angles of cell division in late anaphase to telophase of basal epidermal cells at E16.5 of K14Cre+; Sas-4 $4^{f / w} ; p 53^{f / f}(\mathbf{a})$ and $K 14-C r e+; \operatorname{Sas}-4^{f / f}(\mathbf{b})$ animals. Percentages of parallel $\left(0-29^{\circ}\right)$, oblique $\left(30-59^{\circ}\right)$ and perpendicular $\left(60-90^{\circ}\right)$ divisions (right) of Controls, K14-Cre+; Sas $4^{f / w}$; $p 53^{f / f}$, K14-Cre+; Sas-4 $4^{f / f} ;$ p53 $3^{f / f}(\mathbf{a})$, or Control and K14-Cre+; Sas-4 $4^{f / f}$ (b) animals. Control and K14-Cre+; Sas-4ff; $p 53^{f / f}$ are parts of the same experiment shown in Fig. 4c. (c) Quantification of the density (cells per $100 \mu \mathrm{m}$ ) of basal and suprabasal layer cells of back-skin sections of the epidermis of Control, K14-Cre+; Sas-4 ${ }^{f / w} ; p 53^{f / f}$, K14-Cre+; Sas-4 ${ }^{f / f} ; p 53^{f / f}$ and K14-Cre+; Ift88 $8^{f / f}$ mice at E16.5. Control and K14-Cre+; Sas-4 ${ }^{f / f} ; p 53^{f / f}$ are parts of the same experiment shown in Fig. 4g,h. (d) Quantification of the average cell size of basal and suprabasal layer cells in (c). Control and K14-Cre+; Sas-4 $4^{f / f} ; p 53^{f / f}$ are part of the same experiment shown in Fig. 4i,j. (e) Quantification of the percentage of the EdU-positive cells in the basal layer of back-skin epidermal sections of Control, K14-Cre+; Sas-4 ${ }^{f / w} ; p 53^{f / f}, K 14-C r e+;$ Sas- $4^{f / f} ; p 53^{f / f}$ and K14Cre+; Ift88 ${ }^{f / f}$ mice at E16.5. n. s. = not significant, ${ }^{*} p<0.05,{ }^{* *} p<0.01,{ }^{* * *} p<0.001$ (two-tailed student's T-test). Bars represent mean \pm SD.

Figure S5. Methods to culture, study and image skin explants. (a,b) Immunostaining for pHH3, EdU, and K10 (a) or Cl.CASP3 (b) of Control and MMC-treated flat skin explants on filters at the indicated stages (scale bars: $50 \mu \mathrm{m}$ ). Dashed line represents the epidermal-dermal interface in all panels. (c) Quantification of the K10-positive layer thickness in the Control and MMC-treated skin explants. (d,e) Quantification of the density (cells per $100 \mu \mathrm{m}$ ) of basal (d) and suprabasal (e) layer cells of Control and MMC-treated skin explants. n.s. = not significant, * $p<0.05,{ }^{* *} p<0.01,{ }^{* * *} p<0.001,{ }^{* * * *} p<0.0001$ (two-tailed student's T-test or one-way ANOVA and Tukey's multiple comparisons test). Bars represent mean \pm SD. (f) Representative image of the CAG::H2B-EGFP reporter taken from the time-lapses of flat skin explants at E14.5 shown in the $X Y$ (yellow lines) and $Z$ dimensions (scale bars: $10 \mu \mathrm{m}$ ). (g,h) Immunostaining of the skin explant rolls $(\mathbf{g})$, used for time-lapse imaging, and the flat skin explants (h) at E13.5- 
E15.5, for ITGA6 and K10 (scale bars: $50 \mu \mathrm{m}$ ). (i,j) Immunostaining of back-skin sections at E16.5 for the basement membrane marker COL4A4 (i) or LAMA1 (j) with ITGA6 and K10 (scale bars: $15 \mu \mathrm{m})$.

Figure S6. Measurements of tissue growth, cell densities and mitotic indices in the different axes. (a,b) Quantification of the inter-limb distance (a) and orthogonal distance (b) as depicted in the embryo schematics (red lines) of wild-type embryos from E12.5-E18.5. (c,d) Cell densities (cells per $100 \mu \mathrm{m}$ ) of basal and suprabasal layer cells from transverse back-skin sections of the epidermis between E12.5-E18.5 $(n=5)$. (e) Quantification of pHH3-positive basal (ITGA6) and suprabasal (K10) cells of sagittal and transverse back-skin sections of the epidermis between E12.5-E18.5 $(n=5)$, with examples of the immunostaining shown at E14.5 (arrowheads, scale bar: $10 \mu \mathrm{m}$ ). ${ }^{*} p<0.05,{ }^{* *} p<0.01,{ }^{* * * *} p<0.0001$ (two-tailed student's Ttest). Bars represent mean $\pm S D$.

\section{Movie S1. An example of a perpendicular division orientation with the daughter cell} pointing away from the basement membrane remaining within the basal layer. Time-lapse imaging of the CAG::H2B-EGFP nuclear reporter in E13.5-E14.5 skin-roll explants with frames acquired every 7 minutes. The yellow dots highlight a perpendicular dividing basal cell and its daughter cells (scale bar: $10 \mu \mathrm{m}$ ). This example is representative of 44 out of 94 observed cells from 5 independent experiments (part of experiment shown in Fig. 5a).

\section{Movie S2. An example of a perpendicular division orientation with the daughter cell} pointing away from the basement membrane lingering in a "second" layer. Time-lapse imaging of the CAG::H2B-EGFP nuclear reporter in E13.5-E14.5 skin-roll explants with frames acquired every 7 minutes. The yellow dots highlight a perpendicular dividing basal cell and its 
daughter cells (scale bar: $10 \mu \mathrm{m}$ ). This example is representative of 50 out of 94 observed cells from 5 independent experiments (part of experiment shown in Fig. 5b).

\section{Movie S3. An example of a delaminating basal cell that divides in the suprabasal layers.}

Time-lapse video of the CAG::H2B-EGFP nuclear reporter in E13.5-E14.5 skin-roll explants with frames acquired every 7 minutes shown in greyscale. The yellow dot highlights a basal progenitor which delaminates from the basal layer upwards and subsequently divides. The yellow dots mark the delaminating cell and its progeny (scale bar: $10 \mu \mathrm{m}$ ). This example is representative of a total of 46 cells from 5 independent experiments (part of experiment shown in Fig. 5c).

Table S1. Differentially expressed genes in the centrosome mutant epidermis at E13.5. List of differentially expressed genes with $\geq 2$-fold changes and $p$-values $<0.05$ based on RNASeq analysis of Control and K14-Cre+; Sas- $4^{f / f}$ epidermis at E13.5. The positive values are upregulated in the mutant.

Table S2. Differentially expressed genes in the centrosome mutant keratinocytes at P0. List of differentially expressed genes with $\geq 2$-fold changes and $p$-values $<0.05$ based on RNASeq analysis of Control and K14-Cre+; Sas $-4^{f / f}$ epidermal keratinocytes at P0. The positive values are upregulated in the mutant. 


\section{References}

1 Byrne, C., Hardman, M. \& Nield, K. Covering the limb--formation of the integument. Journal of anatomy 202, 113-124, doi:10.1046/J.1469-7580.2003.00142.X (2003).

2 Smart, I. H. Variation in the plane of cell cleavage during the process of stratification in the mouse epidermis. Br J Dermatol 82, 276-282, doi:10.1111/j.13652133.1970.tb12437.x (1970).

3 Koster, M. I. \& Roop, D. R. The role of p63 in development and differentiation of the epidermis. J Dermatol Sci 34, 3-9, doi:10.1016/j.jdermsci.2003.10.003 (2004).

4 Mills, A. A. et al. p63 is a p53 homologue required for limb and epidermal morphogenesis. Nature 398, 708-713, doi:10.1038/19531 (1999).

5 Hammond, N. L., Dixon, J. \& Dixon, M. J. Periderm: Life-cycle and function during orofacial and epidermal development. Semin Cell Dev Biol 91, 75-83, doi:10.1016/j.semcdb.2017.08.021 (2019).

6 Blanpain, C., Lowry, W. E., Pasolli, H. A. \& Fuchs, E. Canonical notch signaling functions as a commitment switch in the epidermal lineage. Genes Dev 20, 3022-3035, doi:10.1101/gad.1477606 (2006).

7 Hardman, M. J., Sisi, P., Banbury, D. N. \& Byrne, C. Patterned acquisition of skin barrier function during development. Development 125, 1541-1552 (1998).

8 Dotto, G. P. Signal transduction pathways controlling the switch between keratinocyte growth and differentiation. Crit Rev Oral Biol Med 10, 442-457, doi:10.1177/10454411990100040201 (1999).

9 Nowak, J. A., Polak, L., Pasolli, H. A. \& Fuchs, E. Hair follicle stem cells are specified and function in early skin morphogenesis. Cell Stem Cell 3, 33-43, doi:10.1016/j.stem.2008.05.009 (2008).

10 Poulson, N. D. \& Lechler, T. Asymmetric cell divisions in the epidermis. Int Rev Cell Mol Biol 295, 199-232, doi:10.1016/B978-0-12-394306-4.00012-5 (2012).

11 Lechler, T. \& Fuchs, E. Asymmetric cell divisions promote stratification and differentiation of mammalian skin. Nature 437, 275-280, doi:10.1038/nature03922 (2005).

12 Williams, S. E., Beronja, S., Pasolli, H. A. \& Fuchs, E. Asymmetric cell divisions promote Notch-dependent epidermal differentiation. Nature 470, 353-358, doi:10.1038/nature09793 (2011).

13 Kulukian, A. et al. Epidermal development, growth control, and homeostasis in the face of centrosome amplification. Proc Natl Acad Sci U S A 112, E6311-6320, doi:10.1073/pnas.1518376112 (2015).

14 Luxenburg, C., Pasolli, H. A., Williams, S. E. \& Fuchs, E. Developmental roles for Srf, cortical cytoskeleton and cell shape in epidermal spindle orientation. Nat Cell Biol 13, 203-214, doi:10.1038/ncb2163 (2011).

15 Niessen, M. T. et al. aPKClambda controls epidermal homeostasis and stem cell fate through regulation of division orientation. J Cell Biol 202, 887-900, doi:10.1083/jcb.201307001 (2013).

16 Poulson, N. D. \& Lechler, T. Robust control of mitotic spindle orientation in the developing epidermis. J Cell Biol 191, 915-922, doi:10.1083/jcb.201008001 (2010).

17 Seldin, L., Muroyama, A. \& Lechler, T. NuMA-microtubule interactions are critical for spindle orientation and the morphogenesis of diverse epidermal structures. Elife $\mathbf{5}$, doi:10.7554/eLife.12504 (2016).

18 Williams, S. E., Ratliff, L. A., Postiglione, M. P., Knoblich, J. A. \& Fuchs, E. Par3-mInsc and Galphai3 cooperate to promote oriented epidermal cell divisions through LGN. Nat Cell Biol 16, 758-769, doi:10.1038/ncb3001 (2014).

19 Xie, W. \& Zhou, J. Regulation of mitotic spindle orientation during epidermal stratification. J Cell Physiol 232, 1634-1639, doi:10.1002/jcp.25750 (2017). 
20 Schatten, H. \& Sun, Q. Y. Functions and dysfunctions of the mammalian centrosome in health, disorders, disease, and aging. Histochem Cell Biol 150, 303-325, doi:10.1007/s00418-018-1698-1 (2018).

21 Carvalho-Santos, Z. et al. Stepwise evolution of the centriole-assembly pathway. Journal of cell science 123, 1414-1426, doi:10.1242/jcs.064931 (2010).

22 Bond, J. et al. A centrosomal mechanism involving CDK5RAP2 and CENPJ controls brain size. Nature Genetics 37, 353-355, doi:10.1038/ng1539 (2005).

23 Bazzi, H. \& Anderson, K. V. Acentriolar mitosis activates a p53-dependent apoptosis pathway in the mouse embryo. Proc Natl Acad Sci U S A 111, E1491-1500, doi:10.1073/pnas.1400568111 (2014).

24 Insolera, R., Bazzi, H., Shao, W., Anderson, K. V. \& Shi, S. H. Cortical neurogenesis in the absence of centrioles. Nat Neurosci 17, 1528-1535, doi:10.1038/nn.3831 (2014).

25 Lambrus, B. G. et al. p53 protects against genome instability following centriole duplication failure. J Cell Biol 210, 63-77, doi:10.1083/jcb.201502089 (2015).

26 Wong, Y. L. et al. Cell biology. Reversible centriole depletion with an inhibitor of Polo-like kinase 4. Science 348, 1155-1160, doi:10.1126/science.aaa5111 (2015).

27 Fong, C. S. et al. 53BP1 and USP28 mediate p53-dependent cell cycle arrest in response to centrosome loss and prolonged mitosis. Elife 5, doi:10.7554/eLife.16270 (2016).

28 Lambrus, B. G. et al. A USP28-53BP1-p53-p21 signaling axis arrests growth after centrosome loss or prolonged mitosis. J Cell Biol 214, 143-153, doi:10.1083/jcb.201604054 (2016).

29 Meitinger, F. et al. 53BP1 and USP28 mediate p53 activation and G1 arrest after centrosome loss or extended mitotic duration. J Cell Biol 214, 155-166, doi:10.1083/jcb.201604081 (2016).

30 Lambrus, B. G. \& Holland, A. J. A New Mode of Mitotic Surveillance. Trends Cell Biol 27, 314-321, doi:10.1016/j.tcb.2017.01.004 (2017).

31 Fish, J. L., Kosodo, Y., Enard, W., Pääbo, S. \& Huttner, W. B. Aspm specifically maintains symmetric proliferative divisions of neuroepithelial cells. Proceedings of the National Academy of Sciences of the United States of America 103, 10438-10443, doi:10.1073/pnas.0604066103 (2006).

32 Haycraft, C. J. et al. Intraflagellar transport is essential for endochondral bone formation. Development 134, 307-316, doi:10.1242/dev.02732 (2007).

33 Croyle, M. J. et al. Role of epidermal primary cilia in the homeostasis of skin and hair follicles. Development 138, 1675-1685, doi:10.1242/dev.060210 (2011).

34 Ezratty, E. J. et al. A role for the primary cilium in Notch signaling and epidermal differentiation during skin development. Cell 145, 1129-1141, doi:10.1016/j.cell.2011.05.030 (2011).

35 Hafner, M. et al. Keratin 14 Cre transgenic mice authenticate keratin 14 as an oocyteexpressed protein. Genesis 38, 176-181, doi:10.1002/gene.20016 (2004).

36 Mikule, K. et al. Loss of centrosome integrity induces p38-p53-p21-dependent G1-S arrest. Nat Cell Biol 9, 160-170 (2007).

37 Sethi, I., Gluck, C., Zhou, H., Buck, M. J. \& Sinha, S. Evolutionary re-wiring of p63 and the epigenomic regulatory landscape in keratinocytes and its potential implications on species-specific gene expression and phenotypes. Nucleic Acids Res 45, 8208-8224, doi:10.1093/nar/gkx416 (2017).

38 Kashiwagi, M., Kuroki, T. \& Huh, N. Specific inhibition of hair follicle formation by epidermal growth factor in an organ culture of developing mouse skin. Dev Biol 189, 2232, doi:10.1006/dbio.1997.8650 (1997).

39 Gupta, K. et al. Single-Cell Analysis Reveals a Hair Follicle Dermal Niche Molecular Differentiation Trajectory that Begins Prior to Morphogenesis. Dev Cell 48, 17-31.e16, doi:10.1016/j.devcel.2018.11.032 (2019). 
40 Mok, K. W. et al. Dermal Condensate Niche Fate Specification Occurs Prior to Formation and Is Placode Progenitor Dependent. Developmental Cell, doi:10.1016/j.devcel.2018.11.034 (2019).

41 Watt, F. M. \& Fujiwara, H. Cell-extracellular matrix interactions in normal and diseased skin. Cold Spring Harb Perspect Biol 3, doi:10.1101/cshperspect.a005124 (2011).

42 Moriyama, M. et al. Multiple roles of Notch signaling in the regulation of epidermal development. Dev Cell 14, 594-604, doi:10.1016/j.devcel.2008.01.017 (2008).

43 Bata-Csorgo, Z., Hammerberg, C., Voorhees, J. J. \& Cooper, K. D. Flow cytometric identification of proliferative subpopulations within normal human epidermis and the localization of the primary hyperproliferative population in psoriasis. J Exp Med 178, 1271-1281, doi:10.1084/jem.178.4.1271 (1993).

44 Lavker, R. M. \& Sun, T. T. Epidermal stem cells. J Invest Dermato/ 81, 121s-127s, doi:10.1111/1523-1747.ep12540880 (1983).

45 Leigh, I. M., Pulford, K. A., Ramaekers, F. C. \& Lane, E. B. Psoriasis: maintenance of an intact monolayer basal cell differentiation compartment in spite of hyperproliferation. $\mathrm{Br} J$ Dermatol 113, 53-64, doi:10.1111/j.1365-2133.1985.tb02044.x (1985).

$46 \quad$ Kantaputra, P. et al. The smallest teeth in the world are caused by mutations in the PCNT gene. Am J Med Genet A 155A, 1398-1403, doi:10.1002/ajmg.a.33984 (2011).

47 Qvist, P. et al. CtIP Mutations Cause Seckel and Jawad Syndromes. PLoS genetics 7, e1002310, doi:10.1371/journal.pgen.1002310 (2011).

48 Cui, R. et al. Central role of p53 in the suntan response and pathologic hyperpigmentation. Cell 128, 853-864, doi:10.1016/j.cell.2006.12.045 (2007).

49 Goetz, S. C. \& Anderson, K. V. The primary cilium: a signalling centre during vertebrate development. Nat Rev Genet 11, 331-344, doi:nrg2774 [pii]

10.1038/nrg2774 (2010).

50 Pan, Y. et al. gamma-secretase functions through Notch signaling to maintain skin appendages but is not required for their patterning or initial morphogenesis. Dev Cell 7, 731-743, doi:10.1016/j.devcel.2004.09.014 (2004).

51 Box, N. F. \& Terzian, T. The role of p53 in pigmentation, tanning and melanoma. Pigment Cell Melanoma Res 21, 525-533, doi:10.1111/j.1755-148X.2008.00495.x (2008).

52 Mesa, K. R. et al. Homeostatic Epidermal Stem Cell Self-Renewal Is Driven by Local Differentiation. Cell Stem Cell 23, 677-686.e674, doi:10.1016/j.stem.2018.09.005 (2018).

53 Miroshnikova, Y. A. et al. Adhesion forces and cortical tension couple cell proliferation and differentiation to drive epidermal stratification. Nat Cell Biol 20, 69-80, doi:10.1038/s41556-017-0005-z (2018).

54 Nekrasova, O. et al. Desmosomal cadherin association with Tctex-1 and cortactin-Arp2/3 drives perijunctional actin polymerization to promote keratinocyte delamination. Nat Commun 9, 1053, doi:10.1038/s41467-018-03414-6 (2018).

55 Morrow, A., Underwood, J., Seldin, L., Hinnant, T. \& Lechler, T. Regulated spindle orientation buffers tissue growth in the epidermis. Elife 8, doi:10.7554/eLife.48482 (2019).

56 Yuspa, S. H. \& Harris, C. C. Altered differentiation of mouse epidermal cells treated with retinyl acetate in vitro. Exp Cell Res 86, 95-105, doi:10.1016/0014-4827(74)90653-3 (1974).

57 Clayton, E. et al. A single type of progenitor cell maintains normal epidermis. Nature 446, 185-189, doi:10.1038/nature05574 (2007).

58 Box, K., Joyce, B. W. \& Devenport, D. Epithelial geometry regulates spindle orientation and progenitor fate during formation of the mammalian epidermis. Elife 8, doi:10.7554/eLife.47102 (2019).

59 Williams, S. E. \& Fuchs, E. Oriented divisions, fate decisions. Curr Opin Cell Biol 25, 749-758, doi:10.1016/j.ceb.2013.08.003 (2013). 
60 Lefort, K. \& Dotto, G. P. Notch signaling in the integrated control of keratinocyte growth/differentiation and tumor suppression. Semin Cancer Biol 14, 374-386, doi:10.1016/j.semcancer.2004.04.017 (2004).

61 Watt, F. M., Estrach, S. \& Ambler, C. A. Epidermal Notch signalling: differentiation, cancer and adhesion. Curr Opin Cell Biol 20, 171-179, doi:10.1016/j.ceb.2008.01.010 (2008).

62 Jacks, T. et al. Tumor spectrum analysis in p53-mutant mice. Curr Biol 4, 1-7, doi:10.1016/s0960-9822(00)00002-6 (1994).

63 Hadjantonakis, A. K. \& Papaioannou, V. E. Dynamic in vivo imaging and cell tracking using a histone fluorescent protein fusion in mice. BMC Biotechnol 4, 33 (2004).

64 Rübsam, M. et al. E-cadherin integrates mechanotransduction and EGFR signaling to control junctional tissue polarization and tight junction positioning. Nat Commun 8, 1250 , doi:10.1038/s41467-017-01170-7 (2017).

65 Bazzi, H., Fantauzzo, K. A., Richardson, G. D., Jahoda, C. A. B. \& Christiano, A. M. Transcriptional profiling of developing mouse epidermis reveals novel patterns of coordinated gene expression. Developmental Dynamics 236, 961-970, doi:10.1002/dvdy.21099 (2007).

66 Wagle, P., Nikolic, M. \& Frommolt, P. QuickNGS elevates Next-Generation Sequencing data analysis to a new level of automation. BMC Genomics 16, 487, doi:10.1186/s12864015-1695-x (2015).

67 Hulsen, T., de Vlieg, J. \& Alkema, W. BioVenn - a web application for the comparison and visualization of biological lists using area-proportional Venn diagrams. BMC Genomics 9, 488, doi:10.1186/1471-2164-9-488 (2008).

68 Schaefer, C. F. et al. PID: the Pathway Interaction Database. Nucleic Acids Res 37, D674-679, doi:10.1093/nar/gkn653 (2009).

69 Subramanian, A. et al. Gene set enrichment analysis: a knowledge-based approach for interpreting genome-wide expression profiles. Proc Natl Acad Sci U S A 102, 1554515550, doi:10.1073/pnas.0506580102 (2005).

70 DiTommaso, T. \& Foijer, F. Barrier Function Assay. Bio-protocol 4, e1133, doi:10.21769/BioProtoc.1133 (2014). 
a Control

\section{E15.5}

.

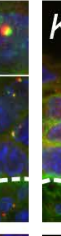

TUंBG

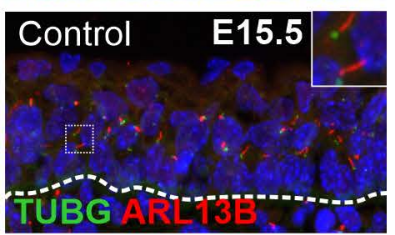

C

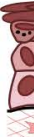

a)

d

$\mathrm{C}$
$\mathrm{E}$
$\mathrm{E}$
$\mathbf{h}$

$=18.5$

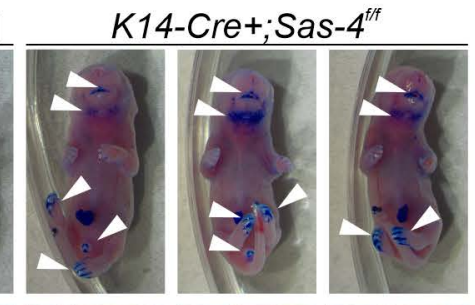

e

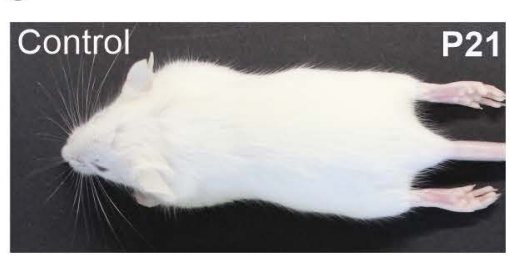

K14-Cre+;Sas-4
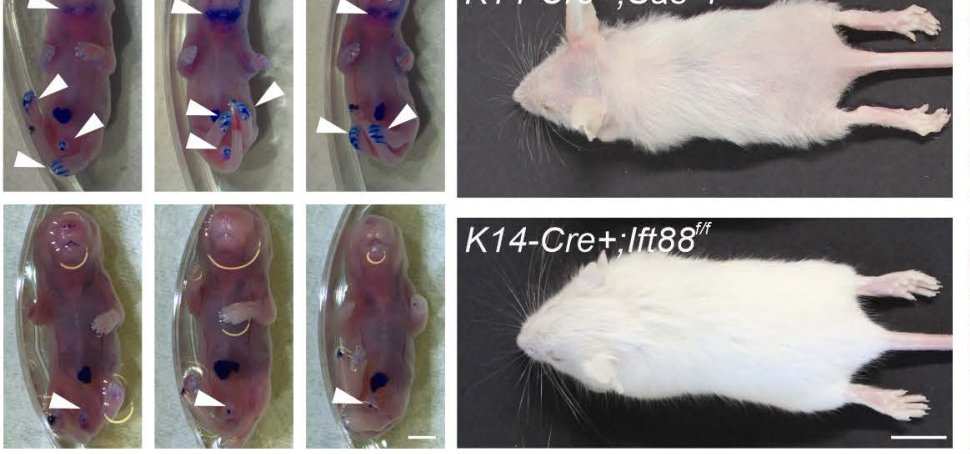

$\tau^{\circ} \quad \begin{aligned} & \text { K14-Cre+;Sas }-4^{\mathrm{f} / \mathrm{f}} \\ & \end{aligned}$

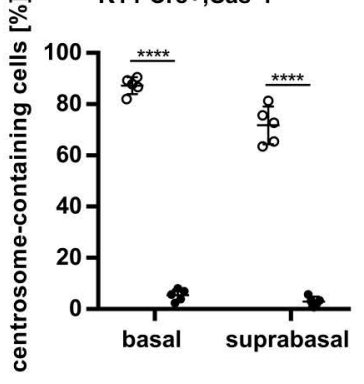

- Control

- K14-Cre+; Ift88 ${ }^{\mathrm{f} / \mathrm{f}}$

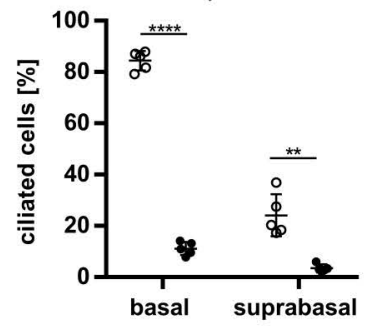

Control

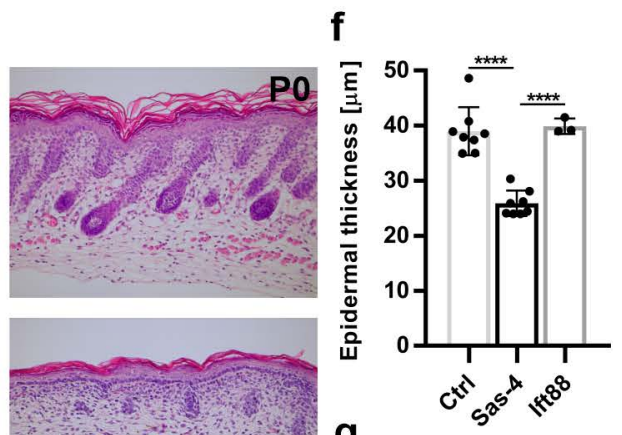

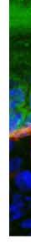

\section{8}

K14-Cre+;Sas-4

$$
\text { K14-Cre+;Ift8 } 8^{f f}
$$

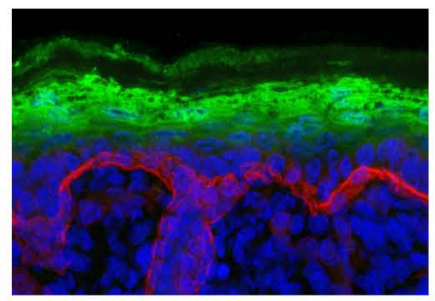




\section{Figure 3} a

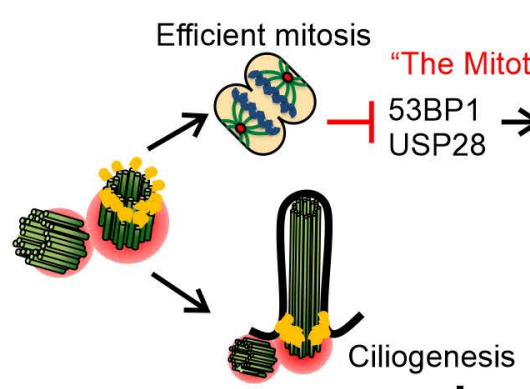

c

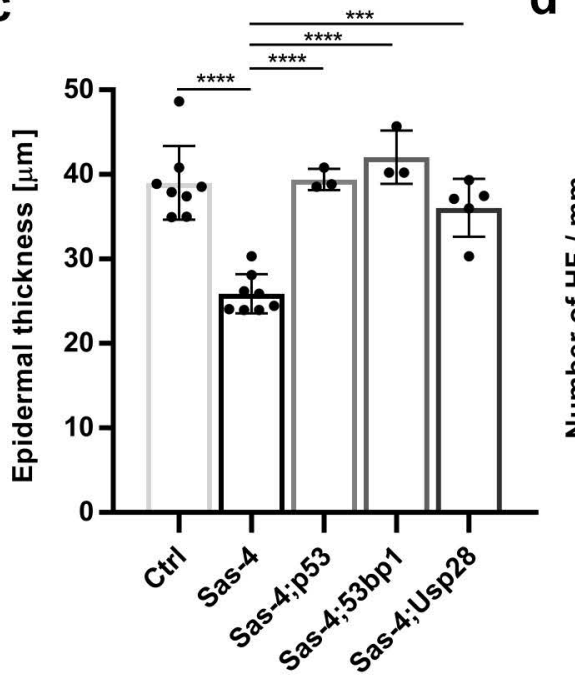

d

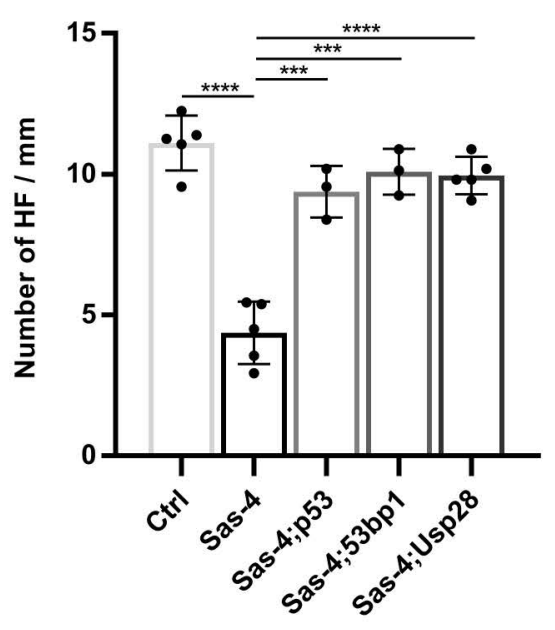

e

Control

Po

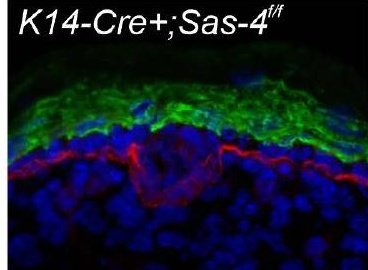

\section{K6A ITGA6}

b
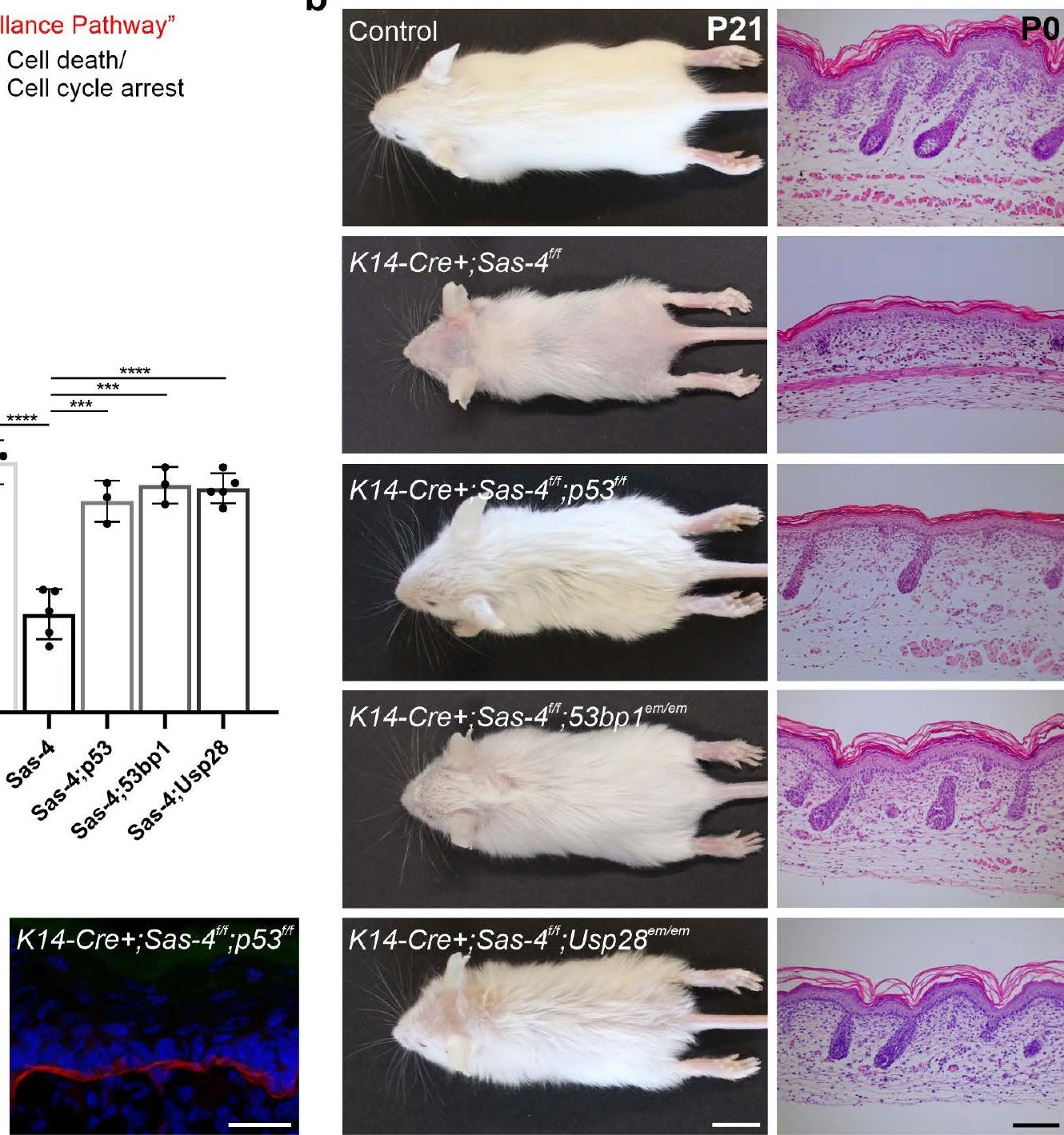
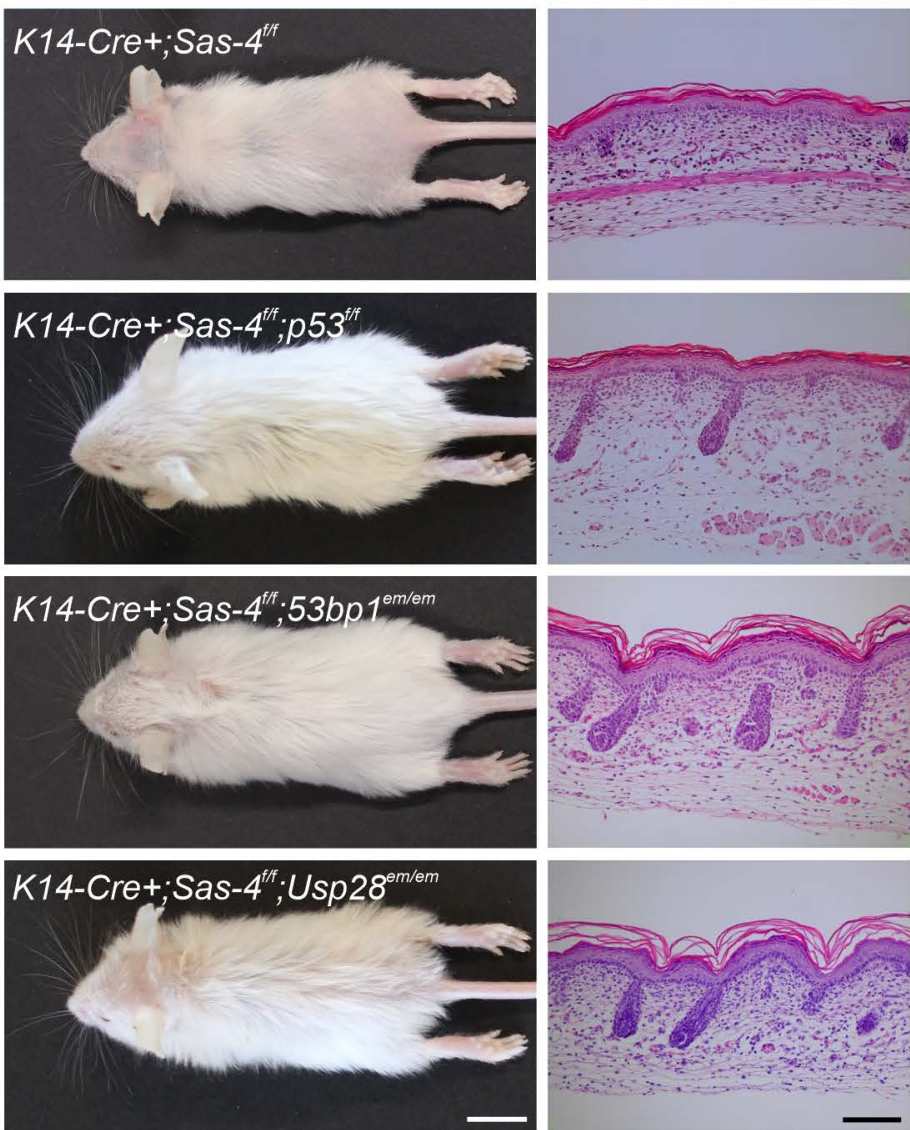
Figure (4hich was not certified by peer review) is the author/funder, who has granted bioRxiv a license to display the preprint in perpet

\section{a parallel}
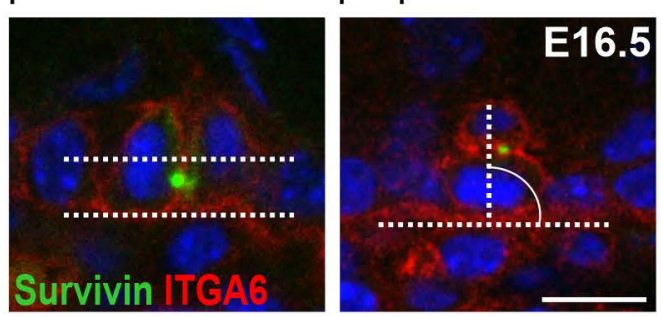
available under aCC-BY-NC-ND 4.0 International license.

\section{b $\mathrm{E} 15.5$}

\section{Control}

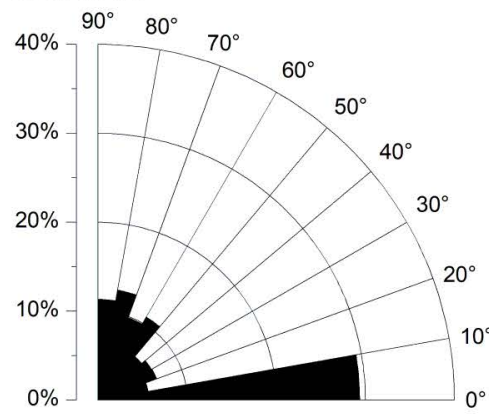

\section{C $\mathbf{E 1 6 . 5}$}

Control

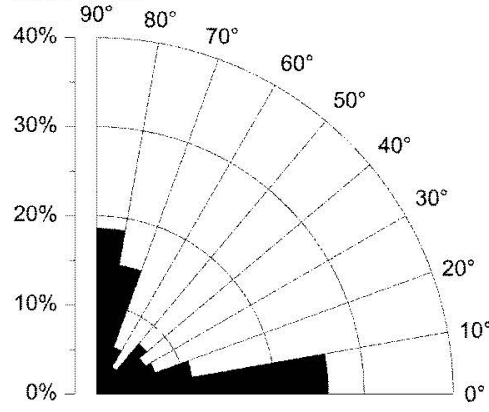

d

\section{E17.5}

\section{Control}

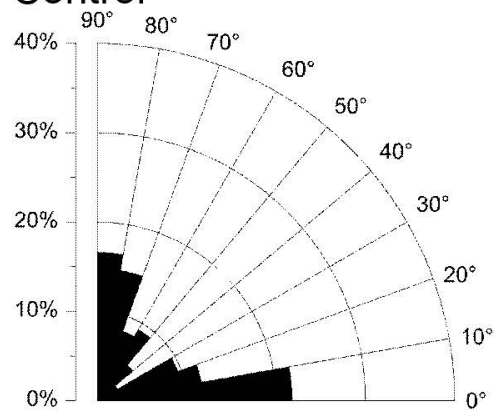

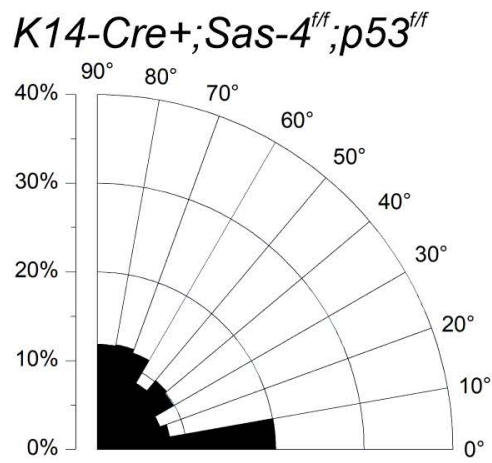

K14-Cre+;Sas- $4^{\text {ff }} ; p 53^{\text {ff }}$

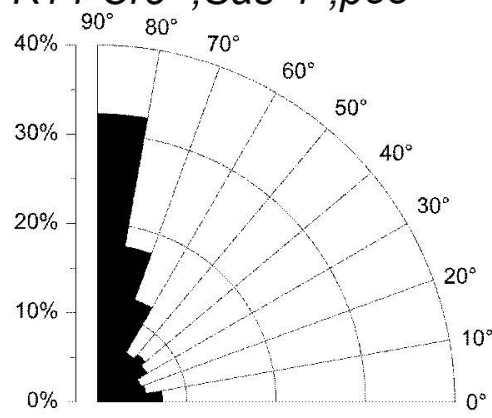

K14-Cre+;Sas- $4^{f f} ; p 53^{f f}$

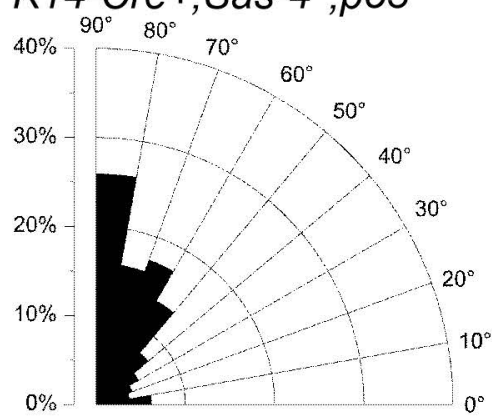

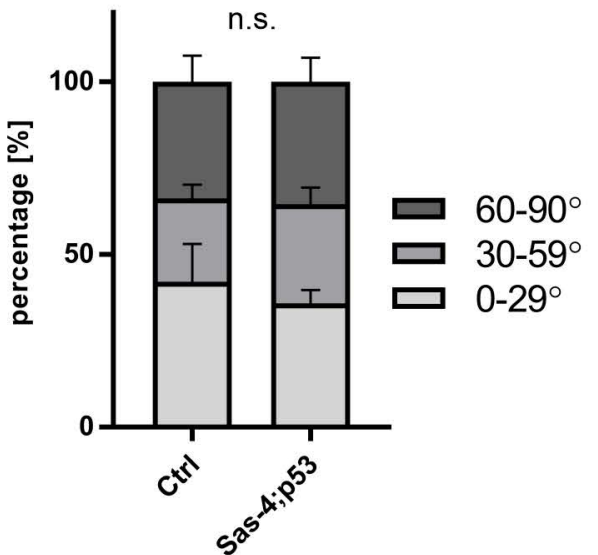
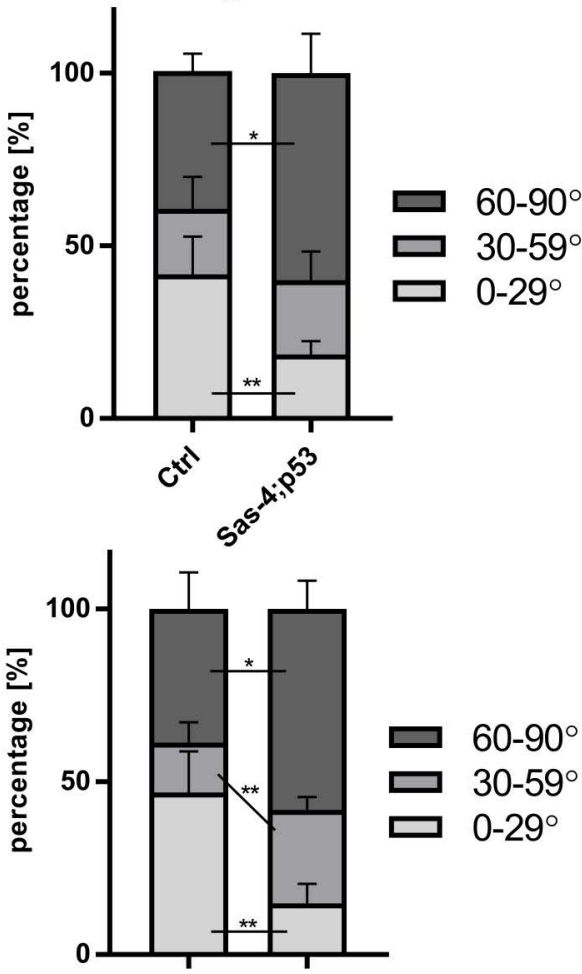

f

\section{.}

g

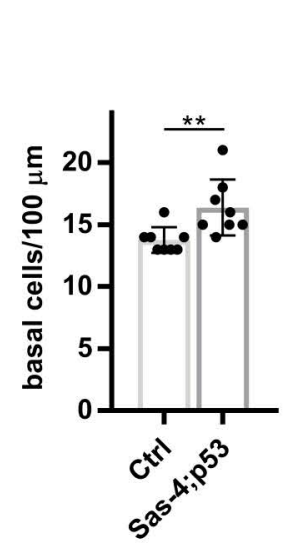

h

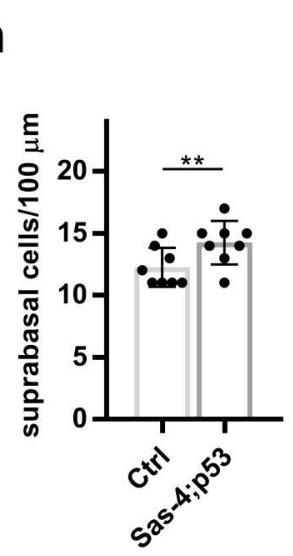

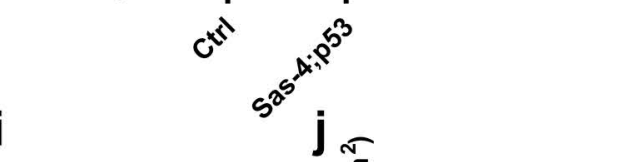
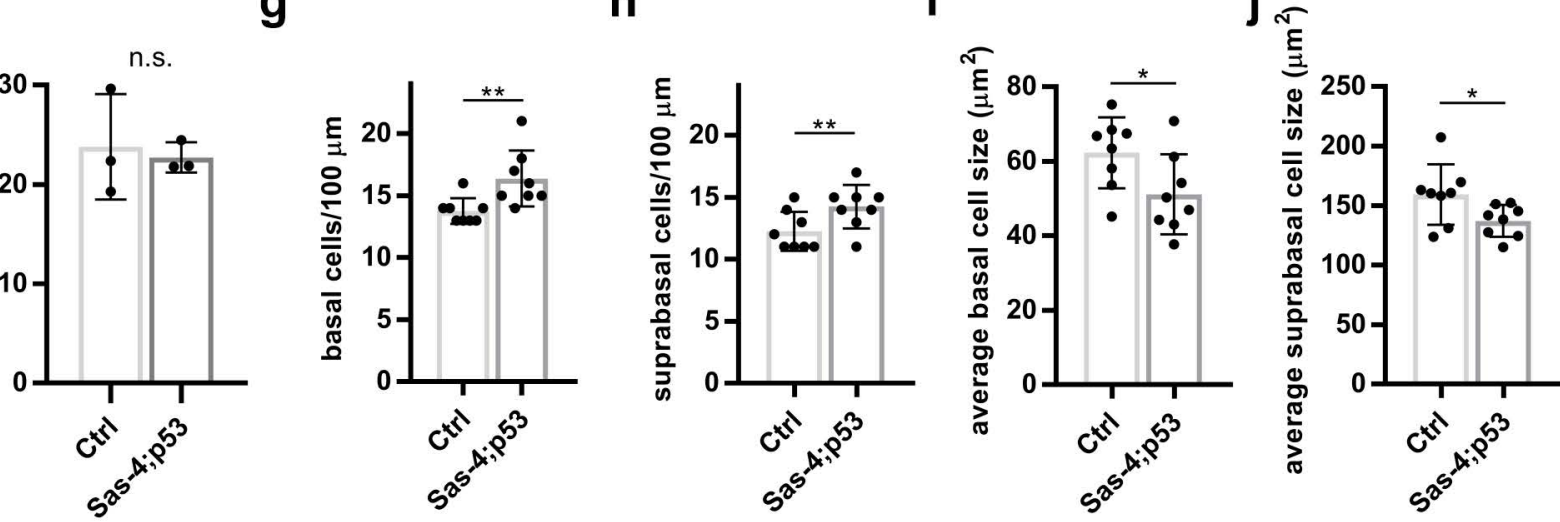
a

\section{CAG::H2B-EGFP'}

\section{-}

0

0

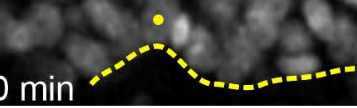

$56 \mathrm{~min}$.

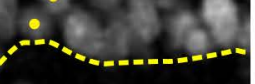

$14 \mathrm{~min}$

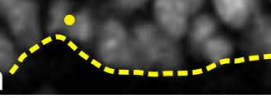

-ro 19.

b

CAG $\because$ H2B-EGFP

$\frac{8}{8}$

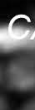

CAG: $H 2 B-E G F P^{60 \mathrm{~m}}$

0

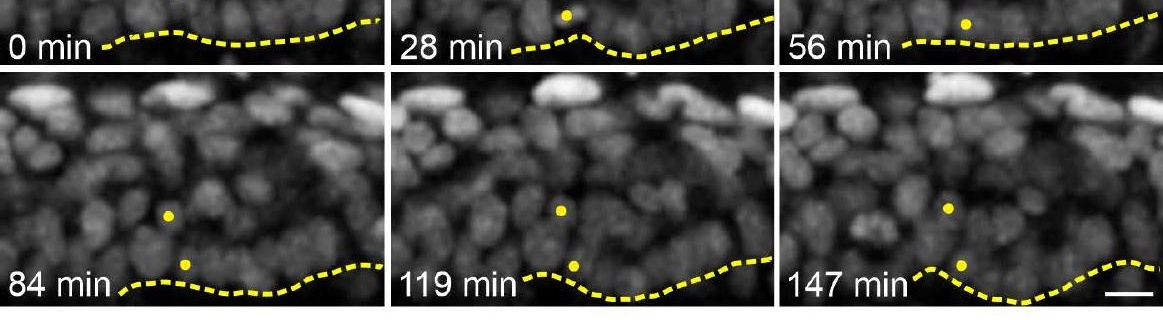

$2 \varepsilon$

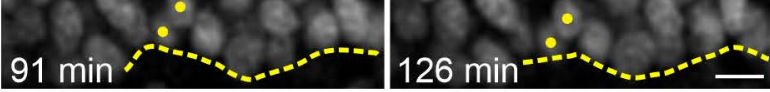

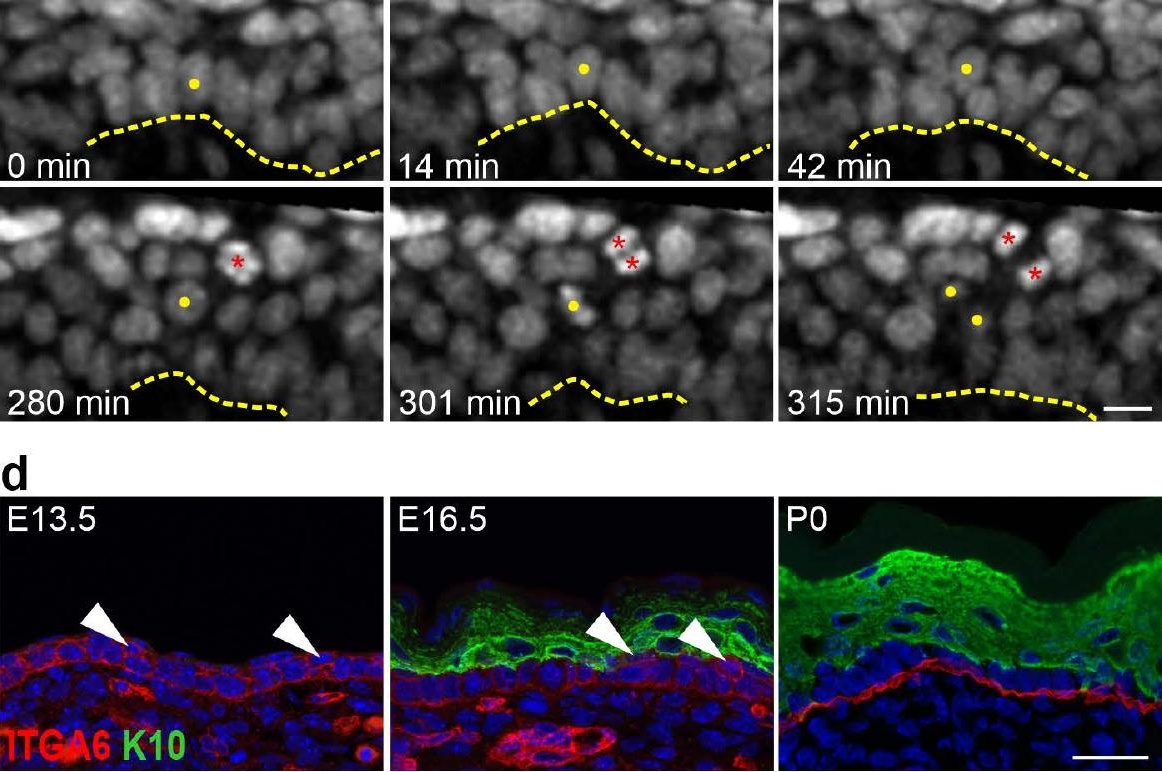




\section{Figure 6}

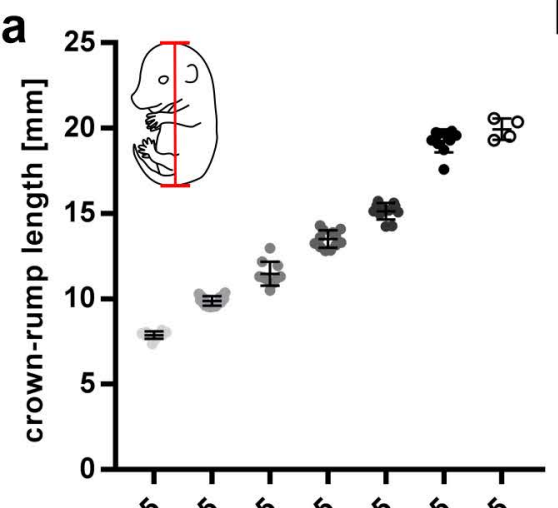

$b_{\text {sagittal }}$

c

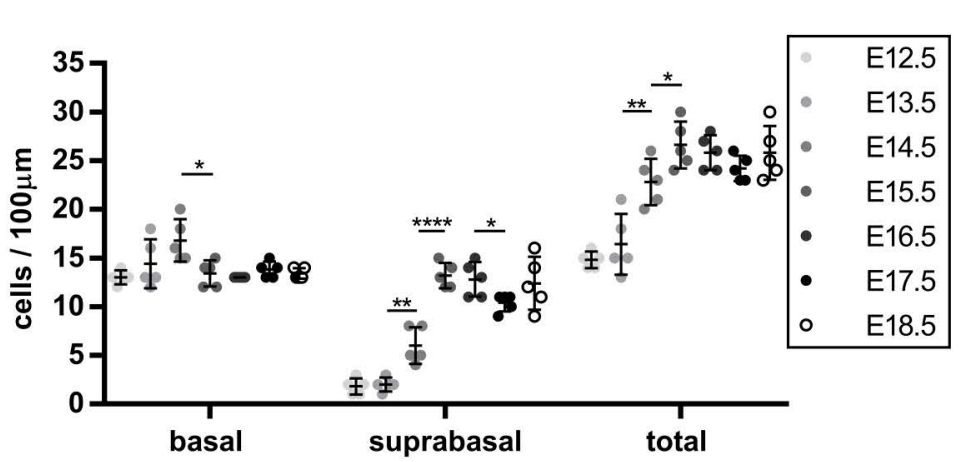

c sagittal

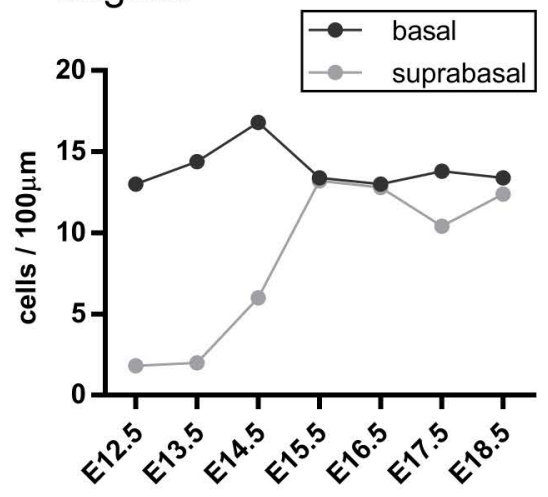

d

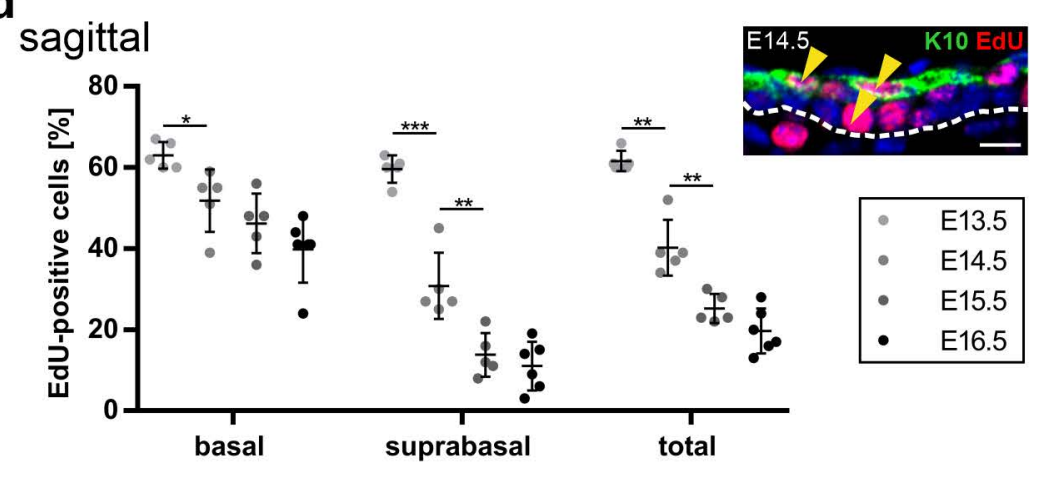

e

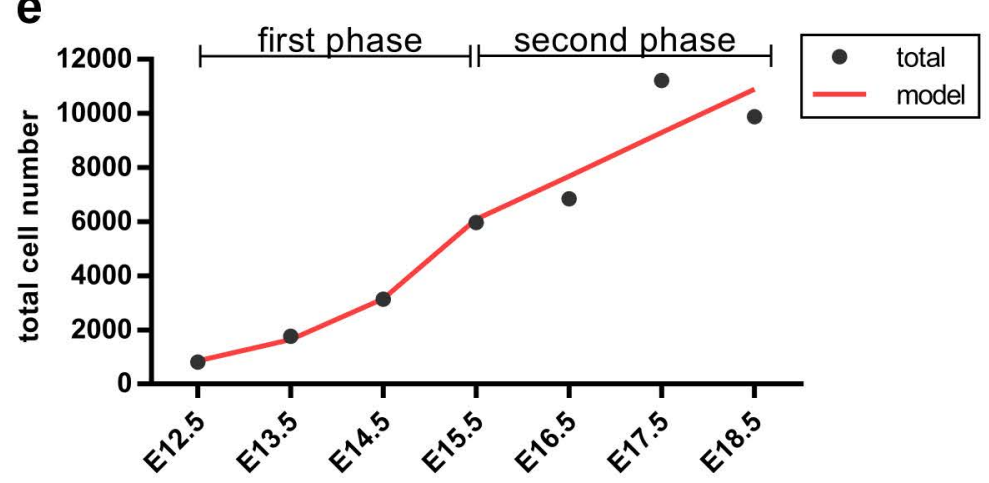




\section{Figure 7}

a

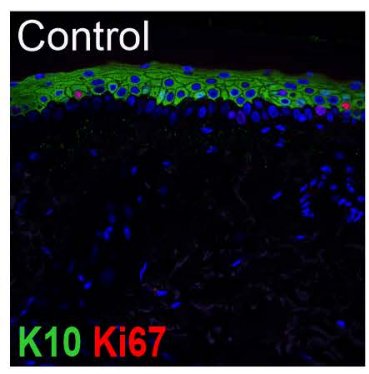

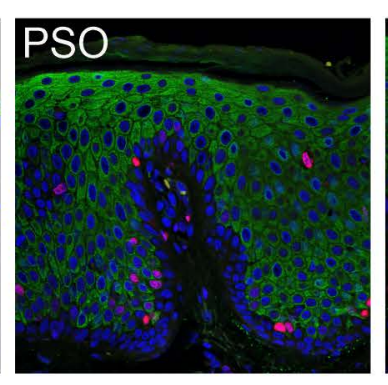

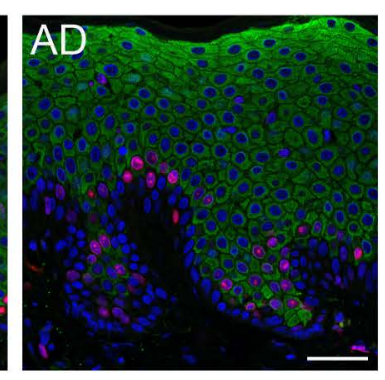

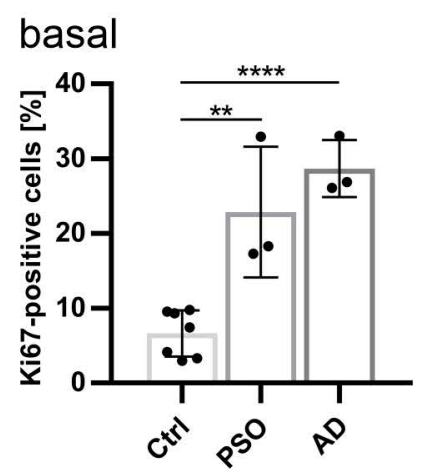

suprabasal

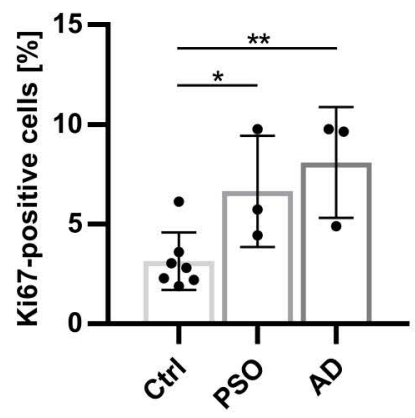

b

$E 9.5-E 12.5$

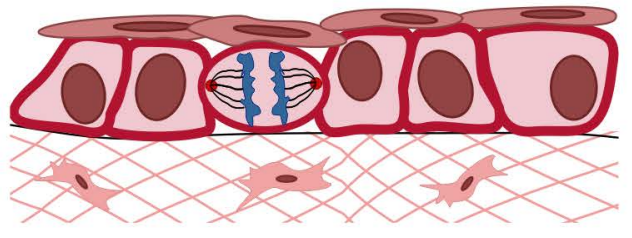

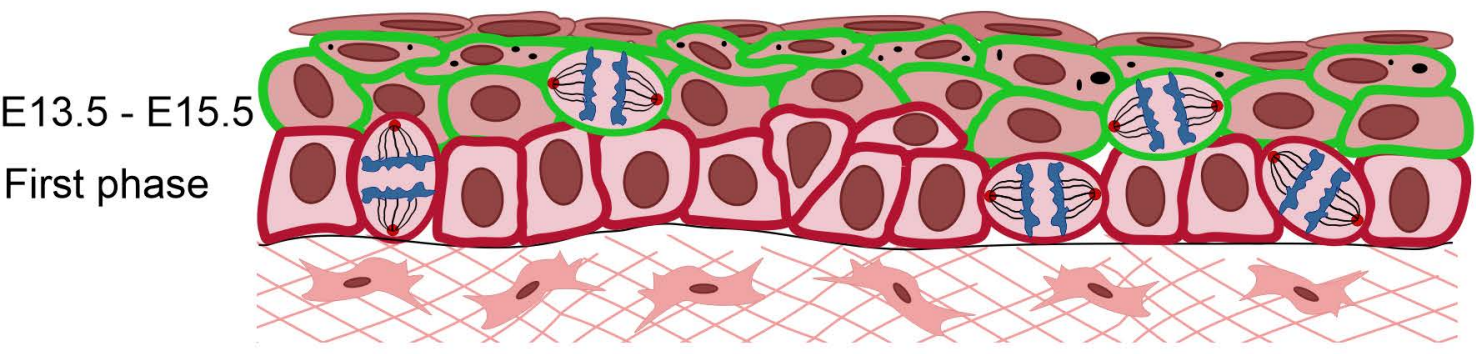

E16.5 - E18.5 Second phase

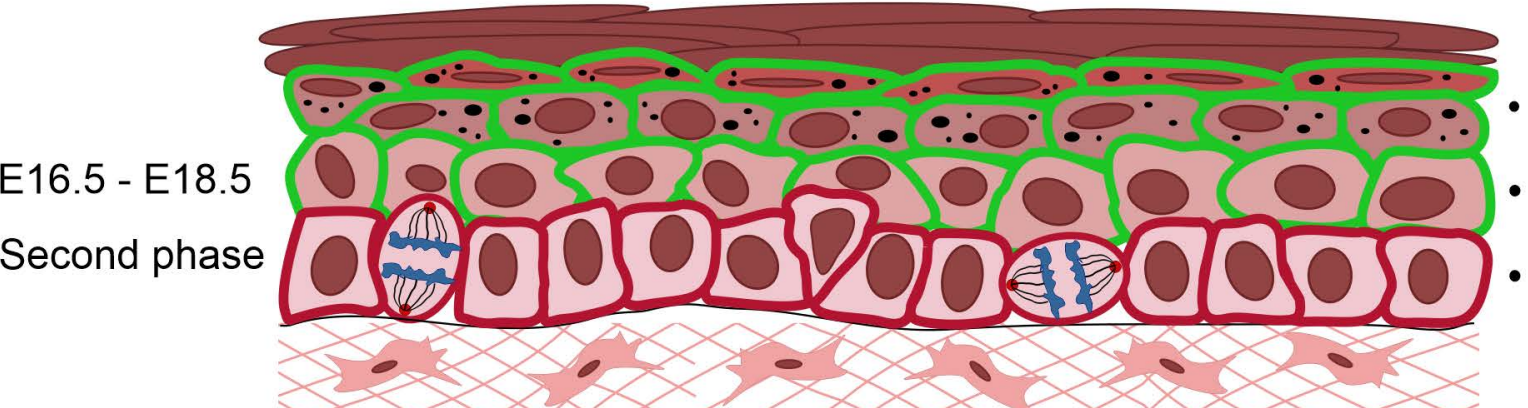

- High proliferation in basal cells

- High proliferation in suprabasal cells

- Delamination

Decrease of proliferation in basal cells

Almost negligible proliferation in suprabasal cells

Delamination? 
a

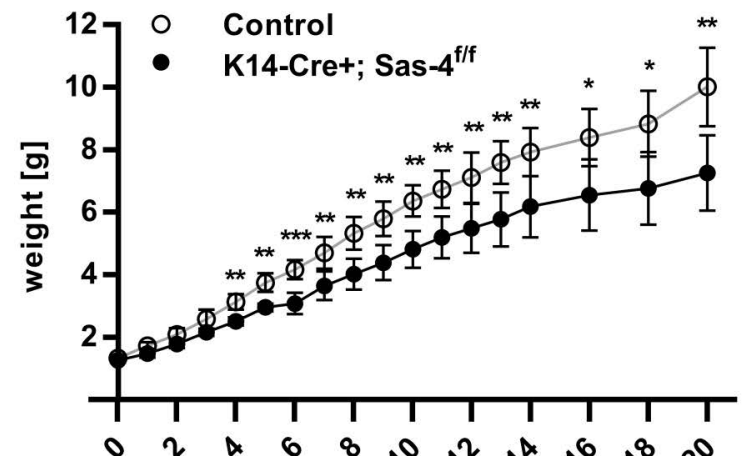
age [days] b

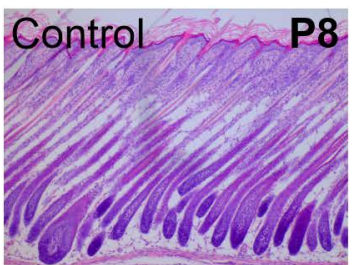

K14-Cre+;Sas-4t

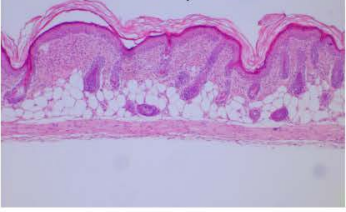

K14-Cre+;/ft8 8
C

Control

P21

K14-Cre+;Sas- $4^{\text {fft }}$

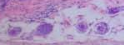

K14-Cre+;/ft8 $8^{8 f t}$ 
Figure S3
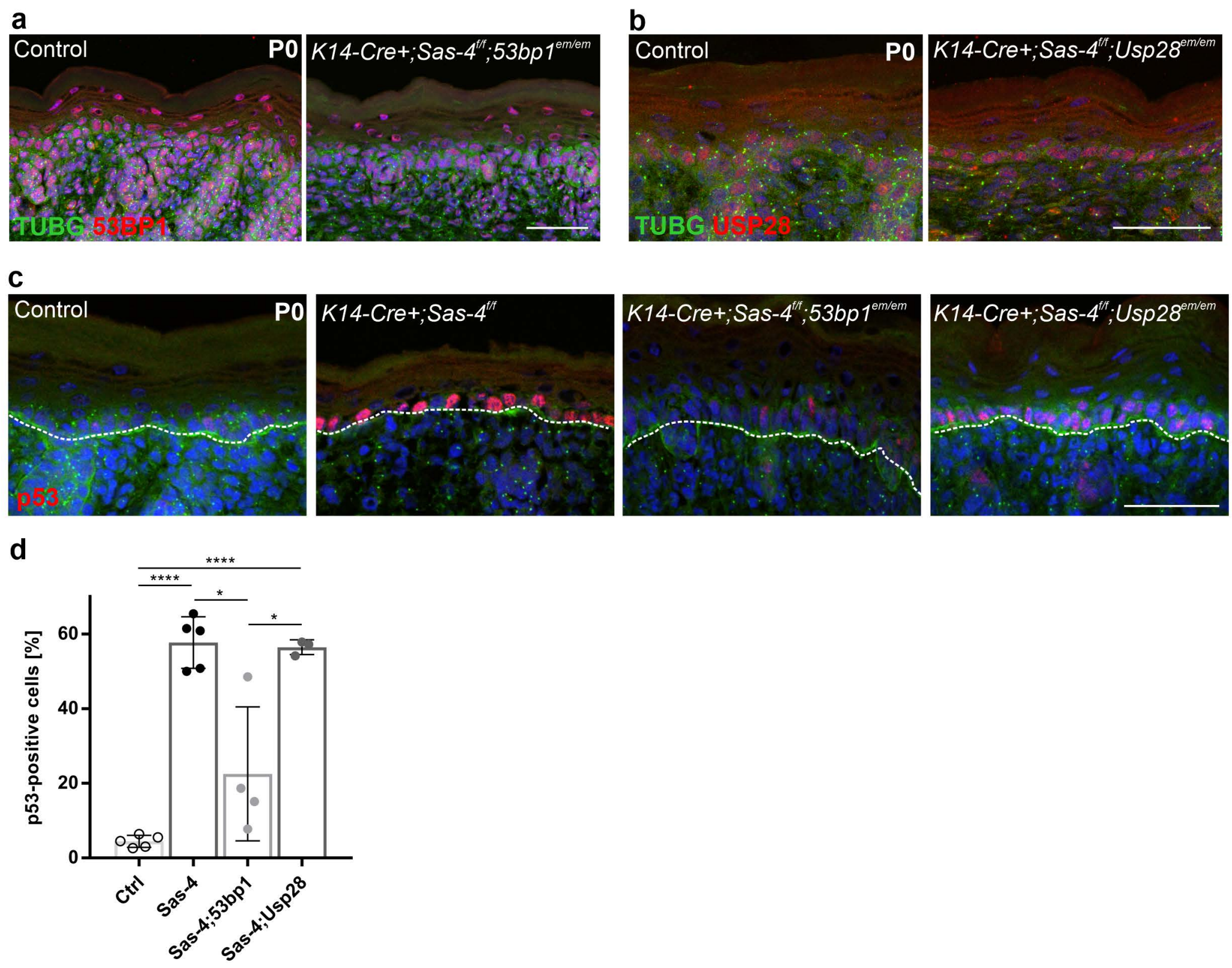


\section{Figure S4}

a

\section{E16.5}

K14-Cre+;Sas $-4^{f / w} ; p 53^{f / f}$

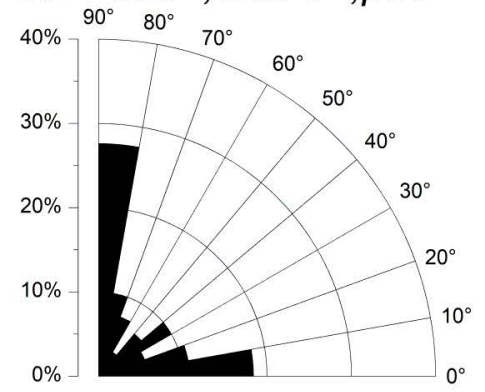

C

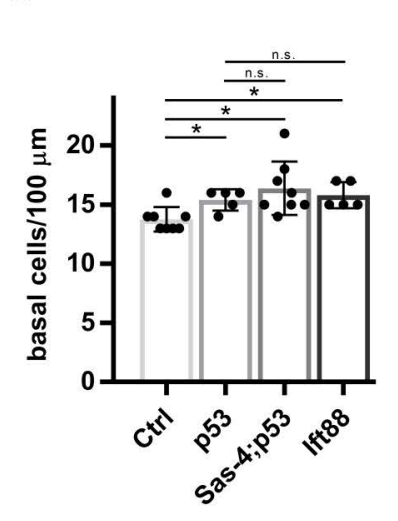

d

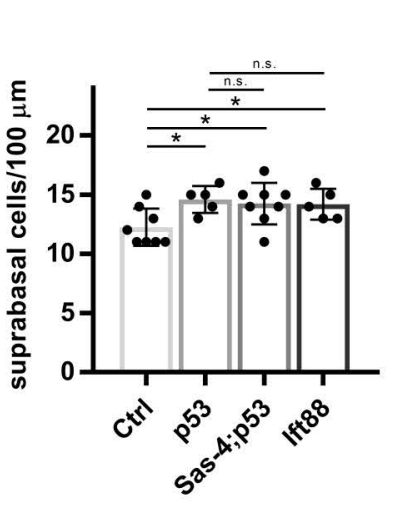

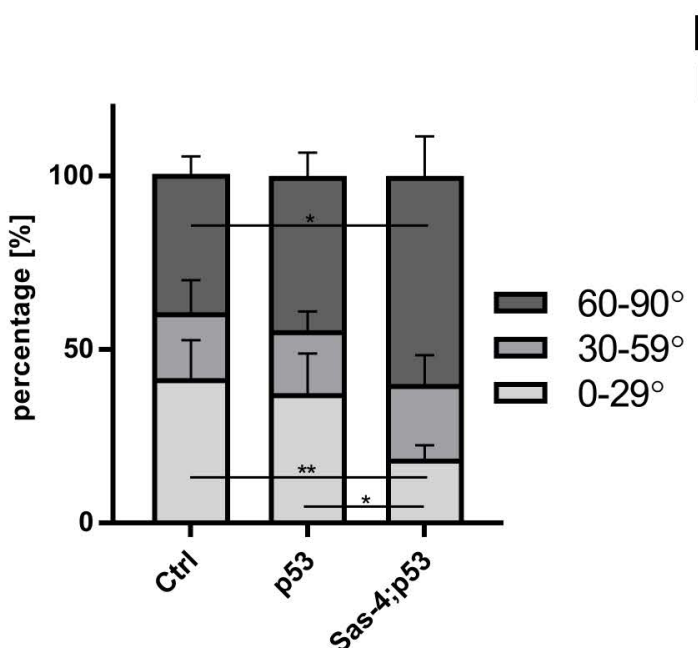

b

\section{E16.5}

K14-Cre+;Sas-4 ${ }^{\text {ff }}$
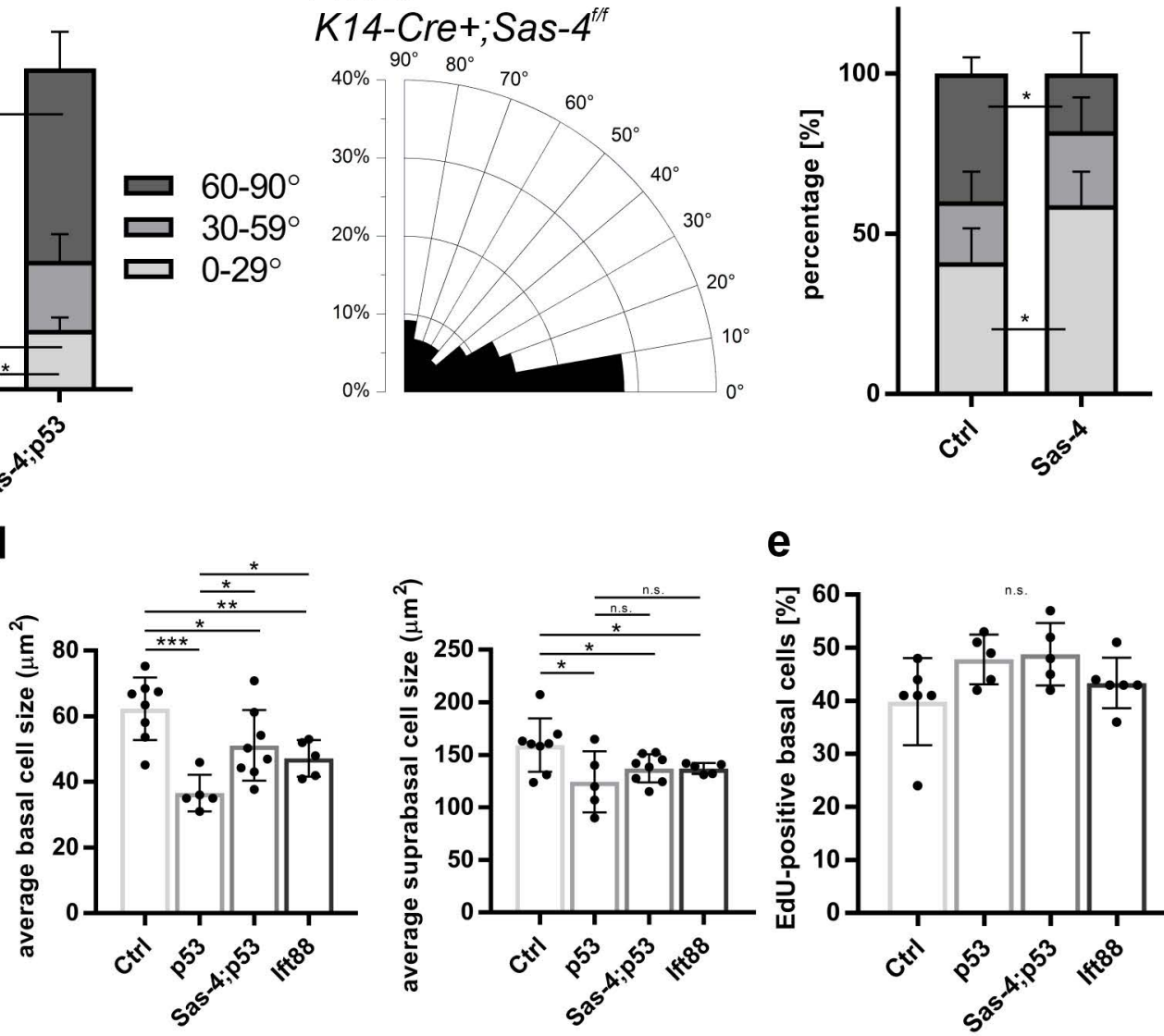

e

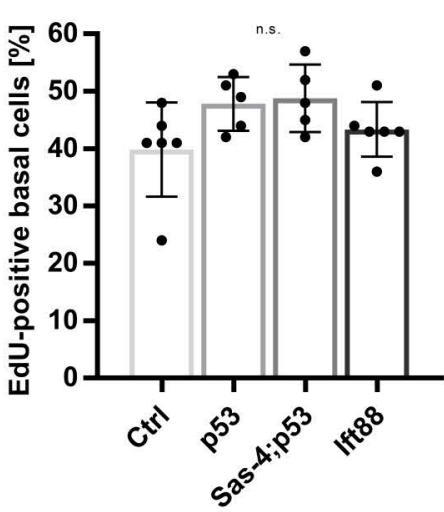



a
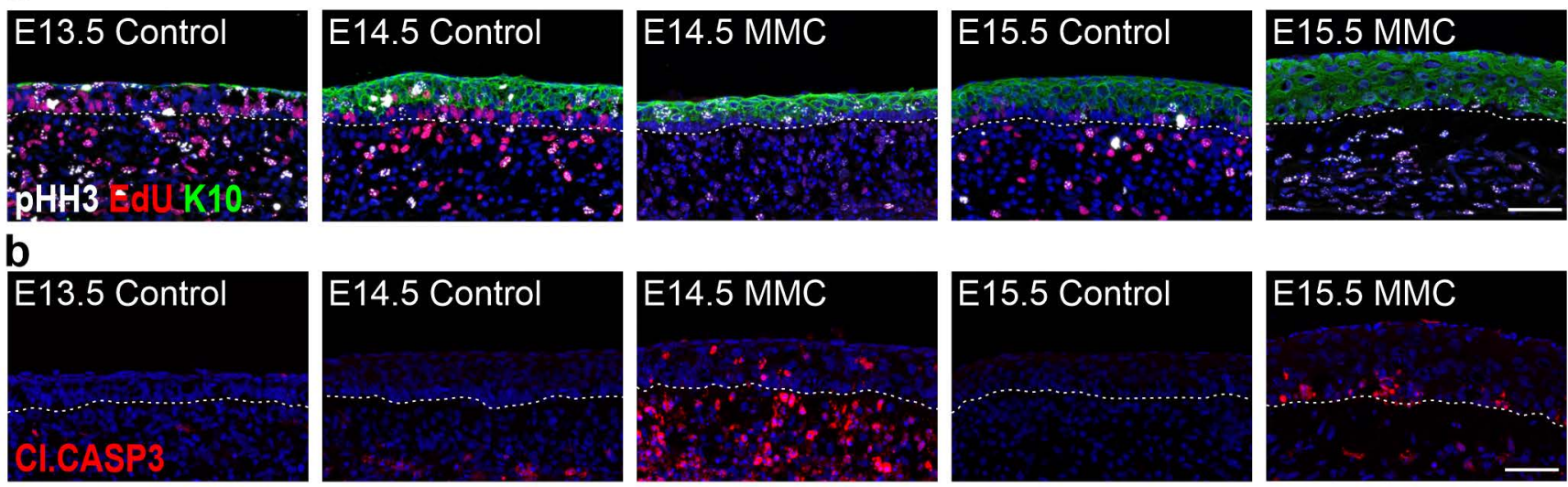

E15.5 MMC
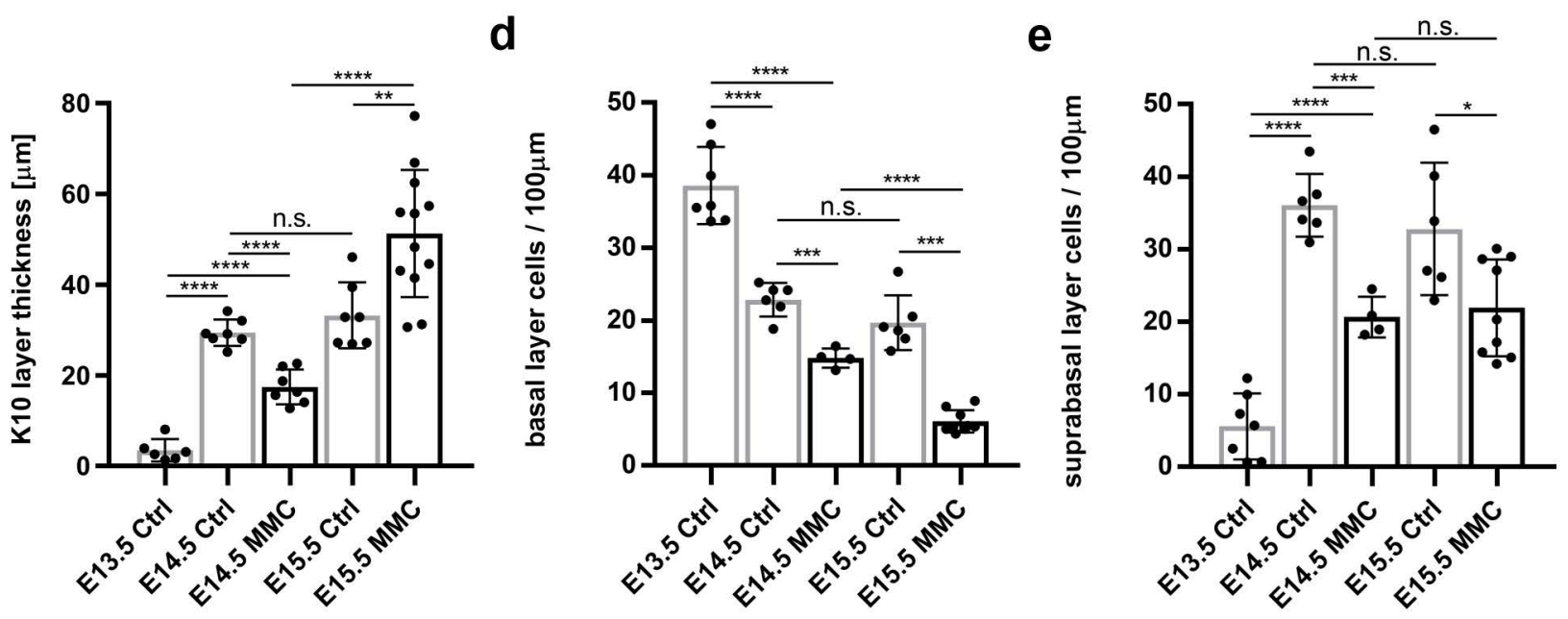

\section{f}

$X Y$

z

g

(

E13.5

K10 ITGA6

E16.5

COL4A4
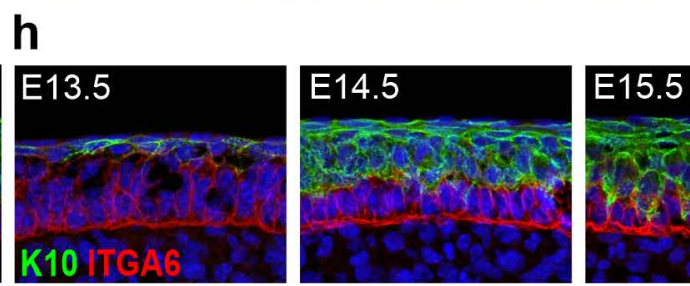

COL4A4 K10 ITGA6

j
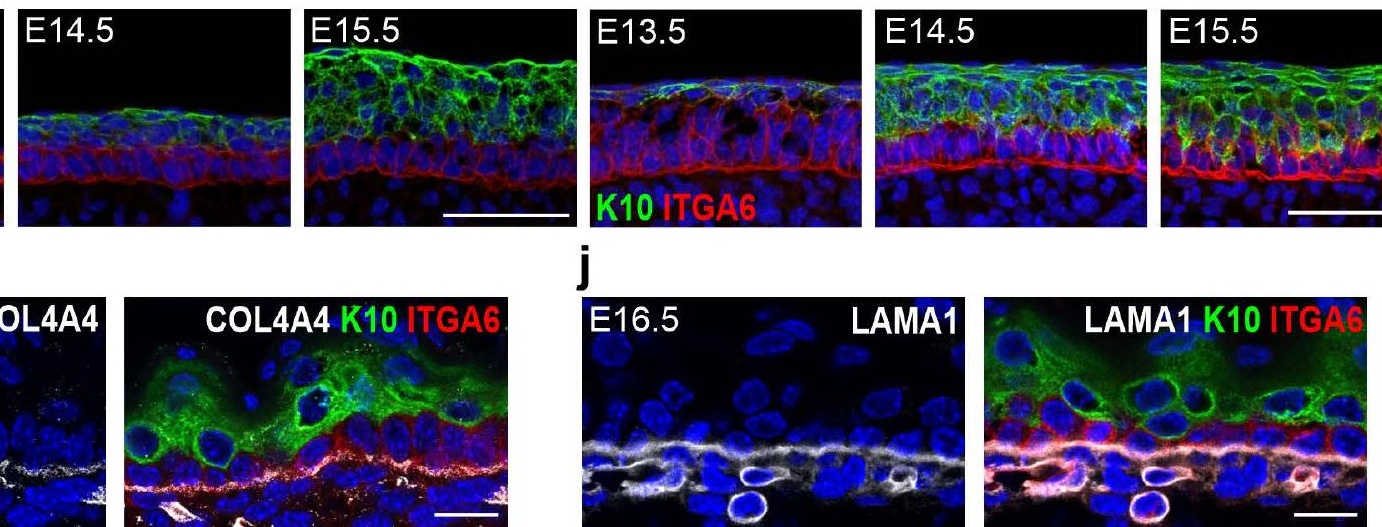


\section{Figure S6}

a

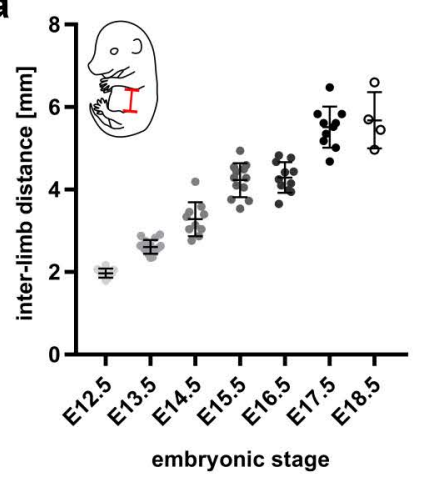

d

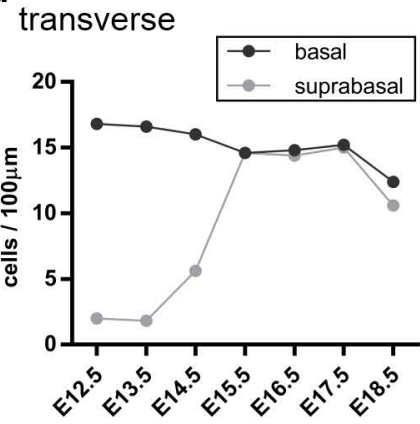

b

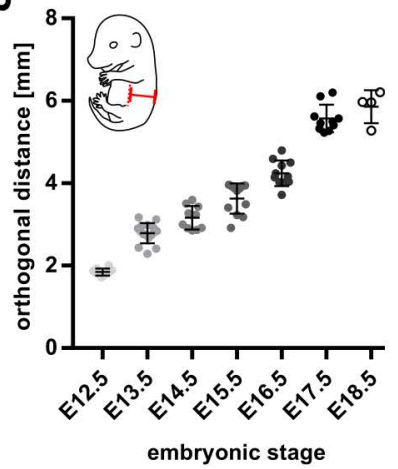

e

sagittal

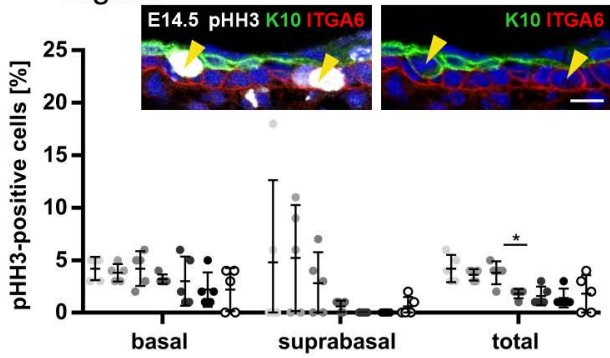

c transverse
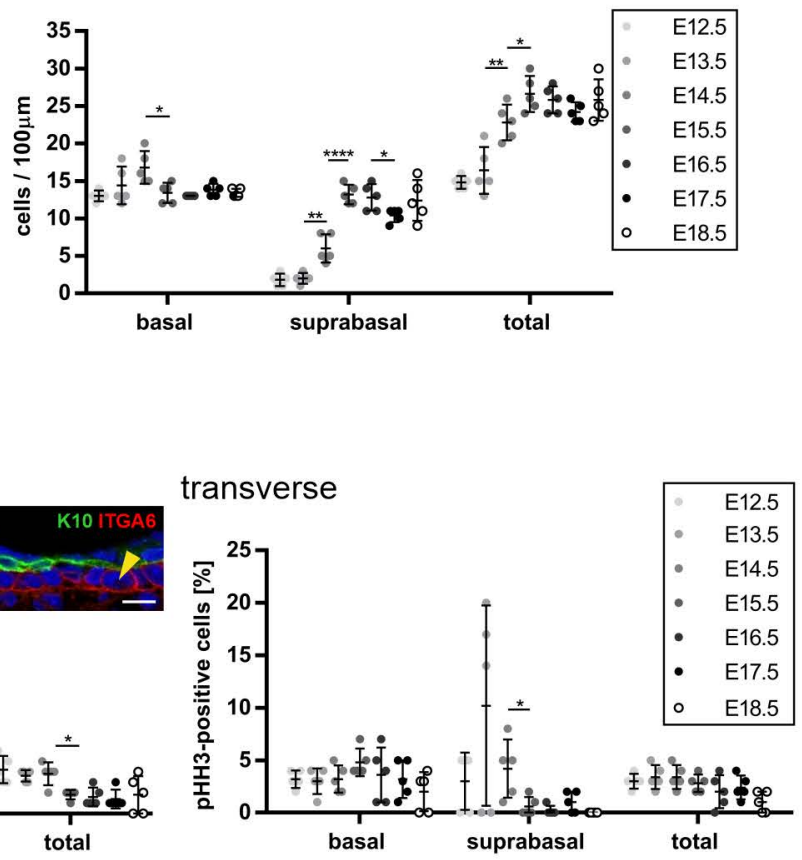